\title{
VERY DEGENERATE ELLIPTIC EQUATIONS UNDER ALMOST CRITICAL SOBOLEV REGULARITY
}

\author{
Albert Clop, Raffaella Giova, Farhad Hatami, Antonia Passarelli di Napoli
}

June 7, 2019

\begin{abstract}
We prove the local Lipschitz continuity and the higher differentiability of local minimizers of functionals of the form

$$
\mathbb{F}(u, \Omega)=\int_{\Omega}(\mathcal{F}(x, D u)+f(x) \cdot u(x)) d x
$$

with non autonomous integrand $\mathcal{F}(x, \xi)$ which is degenerate convex with respect to the gradient variable. The main novelty here is that the results are obtained assuming that the partial map $x \mapsto D_{\xi} \mathcal{F}(x, \xi)$ has weak derivative in the almost critical Zygmund class $L^{n} \log ^{\alpha} L$ and the datum $f$ is assumed to belong to the same Zygmund class.
\end{abstract}

Mathematics Subject Classification. 35J47; 35J70; 49N60.

Key words and phrases. Degenerate convex functionals; Lipschitz regularity; Higher differentiability.

\section{Introduction}

In this paper we deal with the regularity properties of local minimizers of non autonomous and non homogeneous functionals of the form

$$
\mathbb{F}(u, \Omega)=\int_{\Omega}(\mathcal{F}(x, D u)+f(x) \cdot u(x)) d x,
$$

where $\Omega \subset \mathbb{R}^{n}, n \geq 2$ is a bounded open set, $u: \Omega \rightarrow \mathbb{R}^{N}, N \geq 1, \mathcal{F}: \Omega \times \mathbb{R}^{n \times N} \rightarrow[0,+\infty)$ is a Carathéodory function with growth $p \geq 2$, assumed to be uniformly convex with respect to the gradient variable only at $\infty$, and $f: \Omega \subset \mathbb{R}^{n} \rightarrow \mathbb{R}^{N}$ is a given datum. To be more precise, we shall assume that $\xi \mapsto \mathcal{F}(x, \xi)$ is convex and, for an exponent $p \geq 2$, satisfies the following set of hypotheses.

(F0) There exist positive constants $\ell, L$ such that

$$
\ell\left(|\xi|^{p}-1\right) \leq \mathcal{F}(x, \xi) \leq L\left(|\xi|^{p}+1\right)
$$

for a.e. $x \in \Omega$ and every $\xi \in \mathbb{R}^{n \times N}$. 
This is the unique assumption that has to hold true on the whole $\mathbb{R}^{n \times N}$. From now on we list the assumptions that need to hold only at $\infty$, i.e. outside a ball of radius $\mathcal{R}$.

(F1) There exists $F: \bar{\Omega} \times[\mathcal{R}, \infty) \rightarrow[0,+\infty)$ such that

$$
\mathcal{F}(x, \xi)=F(x,|\xi|)
$$

for a.e. $x \in \Omega$ and for every $\xi \in \mathbb{R}^{n \times N} \backslash B_{\mathcal{R}}(0)$.

(F2) The partial map $\xi \mapsto \mathcal{F}(x, \xi)$ is $C^{2}\left(\mathbb{R}^{n \times N} \backslash B_{\mathcal{R}}(0)\right)$ and there exists $\nu>0$ such that

$$
\left\langle D_{\xi \xi} \mathcal{F}(x, \xi) \lambda, \lambda\right\rangle \geqslant \nu|\xi|^{p-2}|\lambda|^{2},
$$

for a.e. $x \in \Omega$, every $\lambda \in \mathbb{R}^{n \times N}$ and every $\xi \in \mathbb{R}^{n \times N} \backslash B_{\mathcal{R}}(0)$.

(F3) There exists a positive constant $L_{1}$ such that

$$
\left|D_{\xi \xi} \mathcal{F}(x, \xi)\right| \leqslant L_{1}|\xi|^{p-2}
$$

for a.e. $x \in \Omega$ and every $\xi \in \mathbb{R}^{n \times N} \backslash B_{\mathcal{R}}(0)$.

(F4) There exist and exponent $\alpha>0$ and a non-negative function $k \in L^{n} \log ^{\alpha} L_{\mathrm{loc}}(\Omega)$ such that

$$
\left|D_{x \xi} \mathcal{F}(x, \xi)\right| \leqslant k(x)|\xi|^{p-1},
$$

for a.e. $x \in \Omega$ and for every $\xi \in \mathbb{R}^{n \times N} \backslash B_{\mathcal{R}}(0)$.

There is no loss of generality in assuming that $\mathcal{R}=1$, what we will do in what follows.

The functionals with degenerate convexity have attracted a wide interest in the last few years. Among the reasons, they arise in problems of optimal transport with congestion effects and in the construction of Wardrop equilibriums in traffic problems (see for instance Carlier, Jiménez and Santambrogio [4). In particular, the regularity of minimizers, and more specifically their Lipschitz continuity, allows the application of the Di Perna - Lions theory to describe the equilibrium configurations. This connection was first described by Brasco, Carlier and Santambrogio [3], and has partially motivated a research line which is nowadays very active.

In this setting, the local Lipschitz regularity of the minimizers was proven in [3, Theorem 5.2] for $f \in C^{\alpha}$ and $\alpha>0$, and in [2, Theorem 2.1] for $f \in L^{s}, s>n$ in case the energy density has the following special structure

$$
\mathcal{F}(x, \xi)=(|\xi|-1)_{+}^{p}, \quad p \geq 2
$$

It is worth mentioning that the regularity of minimizers of non autonomous widely degenerate functionals is challenging also from the theoretical point of view and many contributions to its study are available.

Actually, they fit into the broader contest of asymptotically convex functionals, whose study started with the pioneering paper by Chipot and Evans ([6]) concerning the homogeneous, autonomous, quadratic growth case. Later on, still for the homogeneous, autonomous case, the Lipschitz continuity of local minimizers of asymptotically convex functionals has been established for the superquadratic growth ([25]), in the subquadratic growth case ([31]). Since then, many contributions 
to the regularity theory of asymptotically convex functionals have been established. Among the others we quote the results in [11, 17, 18, 19, 20, 21, 22, 23, 30, 36, 37, 38,

We'd like to recall that, in the non autonomous homogeneous case, i.e. $f \equiv 0$, Fonseca, Fusco and Marcellini proved in [16] that local minimizers of $\mathbb{F}(u, \Omega)$ are Lipschitz continuous if

$$
\left|D_{x \xi} \mathcal{F}(x, \xi)\right| \leq k(x)(1+|\xi|)^{p-1} \quad(x, \xi) \in \Omega \times \mathbb{R}^{n \times N}
$$

for some function $k \in L^{\infty}$, i.e. under a Lipschitz regularity of the partial map $x \mapsto D_{\xi} \mathcal{F}(x, \xi)$. More recently, still for $f \equiv 0$, in [13] this result was extended to the case $k \in L_{\text {loc }}^{s}(\Omega)$ for some $s>n$ (see also [14, 15]).

Especially for applications, it is desirable to understand what is the situation under assumptions on the map $x \mapsto D_{\xi} \mathcal{F}(x, \xi)$ weaker than the Hölder continuity. We know, though, that if $s=n=2$ then $\nabla u \in L^{\infty}$ may fail even in the linear uniformly elliptic setting [8, 33, while in the case $n>2$ some regularity results under a Sobolev or Orlicz Sobolev assumption on the coefficients, that doesn't imply their Hölder continuity, can be found in [24, 26, 27, 28, 32, 34,

The case $k \in L_{\text {loc }}^{n}(\Omega)$ has been studied in [9] for homogeneous functionals with degenerate convexity, but obtaining only $W^{1, t}$ regularity for every $t \in[p, \infty)$.

As far as we know, no Lipschitz regularity results are available for local minimizers of this class of functionals under weaker assumptions on the coefficients and the datum and this motivated our study.

Here we will show that the gradient of the local minimizers of $\mathbb{F}(u, \Omega)$ is locally bounded in $\Omega$ under a regularity assumption on the map $x \rightarrow D_{\xi} f(x, \xi)$ and the datum $f$ that, roughly speaking, will be intermediate between $L^{n}$ and $L^{s}$, with $s>n$.

Actually, we are able to prove that in order to obtain the local Lipschitz regularity of the minimizers it suffices to assume that both the weak derivative with respect to $x$ of $D_{\xi} f(x, \xi)$ and the datum $f$ belong to the Orlicz Zygmund class $L^{n} \log ^{\alpha} L$, with $\alpha>0$ sufficiently large with respect to the dimension $n$. More precisely, our main result is the following

Theorem 1. Let $u \in W_{\text {loc }}^{1, p}(\Omega)$ be a local minimizer of the functional $\mathbb{F}(u, \Omega)$ in (1.1), and assume that the energy density $\mathcal{F}(x, \xi)$ satisfies assumptions (F0)-(F4). If $f \in L^{n} \log ^{\alpha} L_{\operatorname{loc}}(\Omega)$, with $\alpha>3 n$ and where $\alpha$ is the exponent appearing in (F4), then $D u \in L_{\mathrm{loc}}^{\infty}(\Omega)$ and the following estimate

$$
\sup _{B_{\rho}}|D u| \leqslant \hat{C}\left(\int_{B_{r}}(1+|D u|)^{p} d x,\right)^{\frac{1}{p}}
$$

holds for every balls $B_{\rho} \subset B_{r} \Subset \Omega$ and for a constant $\hat{C}=\hat{C}\left(n, p, \alpha, \nu, L_{1}, \rho, r,\|k\|_{L^{n} \log ^{\alpha} L\left(B_{r}\right)},\|f\|_{L^{n} \log ^{\alpha} L\left(B_{r}\right)}\right)$.

Note that, by virtue of the embedding Theorems in the Orlicz-Sobolev setting ([7]), our assumption on the partial map $x \mapsto D_{\xi} \mathcal{F}(x, \xi)$ implies the continuity of the coefficients but not their Hölder continuity.

As usual, the proof of this Theorem is divided in two parts: an a priori $L^{\infty}$ estimate, and an approximation argument.

The a priori estimate is achieved by the use of test functions that vanish on the set where the 
functional in not uniformly convex. The novelty here is the use of a suitable Young's inequality in Orlicz space and an appropriate modification of the Moser iteration argument.

The size of the exponent $\alpha$ in assumption (F4) is used in order to let this iteration argument work. Once the a priori estimate is established, we perform an approximation procedure with a sequence of functionals regular enough to apply the a priori estimate to their minimizers.

The lack of the uniqueness of the minimizers, due to the lack of uniform convexity of the integrand, could effect the approximation argument. We overcome this difficulty, as done in [5, by adding to the approximating functionals a penalization term that forces the approximating sequence to converge to an arbitrarily fixed local minimizers.

It is worth mentioning that in order to start the Moser iteration procedure, we need to estimate quantities involving the second derivatives of the local minimizers. Therefore, another result that we are able to establish here consists in showing that minimizers turn out to gain one degree of differentiability, in the weak sense, away from the degeneracy set. This can be quantitatively stated as follows. Denoting by

$$
\mathcal{G}(t):=1+\int_{0}^{t}(1+s)^{\frac{p-4}{2}} s d s,
$$

we have the following higher differentiability result.

Theorem 2. Let $u \in W^{1, p}\left(\Omega ; \mathbb{R}^{n \times N}\right)$ be a local minimizer of the functional $\mathbb{F}(\cdot, \Omega)$ in (1.1), and assume that the energy density $\mathcal{F}(x, \xi)$ satisfies assumptions (F0)-(F4). If $f \in L^{n} \log ^{\alpha} L_{\mathrm{loc}}(\Omega)$ with $\alpha>3 n$, then

$$
\mathcal{G}\left((|D u|-1)_{+}\right) \in W_{\mathrm{loc}}^{1,2}(\Omega)
$$

and the following Caccioppoli type inequality holds,

$$
\int_{B_{\rho}\left(x_{0}\right)}\left|D\left(\mathcal{G}\left((|D u|-1)_{+}\right)\right)\right|^{2} \leq \widetilde{C} \int_{B_{r}\left(x_{0}\right)}\left(1+|D u|^{p}\right) d x
$$

for every balls $B_{\rho} \subset B_{r}\left(x_{0}\right) \Subset \Omega$, for some $\widetilde{C}=\widetilde{C}\left(n, p, \alpha, \nu, L_{1}, \rho, r,\|k\|_{L^{n} \log ^{\alpha} L\left(B_{r}\right)},\|f\|_{L^{n} \log ^{\alpha} L\left(B_{r}\right)}\right)$.

The proof of Theorem 2 is established as before combining an a priori estimate with an approximation argument.

This time the regularity of the approximating minimizers is transferred to the limit by the use of a measure theory result ( see Proposition 8 below) that is an improved version of Proposition 6 in [10.

Let us mention that in equation (1.3), the term on the left hand side is equivalent to

$$
\int_{B_{\rho}\left(x_{0}\right)}\left|D\left(\mathcal{G}\left((|D u|-1)_{+}\right)\right)\right|^{2}=\int_{B_{\rho}\left(x_{0}\right)}(|D u|-1)_{+}^{2}|D u|^{p-4}\left|D^{2} u\right|^{2} d x
$$

so that the above result is, in fact, a weighted bound for $D^{2} u$ with the weight $(|D u|-1)_{+}^{2}|D u|^{p-4}$.

The paper is structured as follows. In Section 2 we recall some preliminaries. In Section 3 we prove both the interior a priori Lipschitz and the a priori second order Sobolev estimates. In Section 4 we prove the Lipschitz regularity of the minimizers of $\mathbb{F}(u, \Omega)$, i.e. Theorem 1 In Section 5 we prove the interior second order Sobolev regularity of the minimizers of $\mathbb{F}(u, \Omega)$, i.e. Theorem 2 . 


\section{$2 \quad$ Preliminary results}

We will follow the usual convention and denote by $c$ or $C$ a general constant that may vary on different occasions, even within the same line of estimates. Relevant dependencies on parameters and special constants will be suitably emphasized using parentheses or subscripts. All the norms we use will be the standard Euclidean ones and denoted by $|\cdot|$ in all cases. In particular, for matrices $\xi$, $\eta \in \mathbb{R}^{n \times m}$ we write $\langle\xi, \eta\rangle:=\operatorname{trace}\left(\xi^{T} \eta\right)$ for the usual inner product of $\xi$ and $\eta$, and $|\xi|:=\langle\xi, \xi\rangle^{\frac{1}{2}}$ for the corresponding euclidean norm. By $B_{r}(x)$ we will denote the ball in $\mathbb{R}^{n}$ centered at $x$ of radius $r$. The integral mean of a function $u$ over a ball $B_{r}(x)$ will be denoted by $u_{x, r}$, that is

$$
u_{x, r}:=\frac{1}{\left|B_{r}(x)\right|} \int_{B_{r}(x)} u(y) d y
$$

where $\left|B_{r}(x)\right|$ is the Lebesgue measure of the ball in $\mathbb{R}^{n}$. If no confusion arises, we shall omit the dependence on the center.

The following lemma is an important application in the so called hole-filling method. Its proof can be found for example in [29, Lemma 6.1] .

Lemma 3. Let $h:\left[r, R_{0}\right] \rightarrow \mathbb{R}$ be a nonnegative bounded function and $0<\vartheta<1, A, B \geq 0$ and $\beta>0$. Assume that

$$
h(s) \leq \vartheta h(t)+\frac{A}{(t-s)^{\beta}}+B,
$$

for all $r \leq s<t \leq R_{0}$. Then

$$
h(r) \leq \frac{c A}{\left(R_{0}-r\right)^{\beta}}+c B
$$

where $c=c(\vartheta, \beta)>0$.

In what follows, for each $\gamma \geq 0$, we will denote

$$
\Phi(t)=\Phi_{\gamma}(t)=\frac{t^{2}}{(1+t)^{2}}(1+t)^{\gamma} .
$$

For such $\Phi$, one can easily check that

$$
t \Phi^{\prime}(t) \leq 2(1+\gamma) \Phi(t)
$$

and also that, since

$$
\Phi^{\prime}(t) \leq 2(1+\gamma) t\left(1+t^{2}\right)^{\gamma-2}
$$

we have

$$
\Phi^{\prime}(t) \leq 2^{\gamma+1}(1+\gamma) \quad \text { for every } t \in(0,1) .
$$

We introduce the following notation for the positive part of $|D u|-1$,

$$
P=(|D u|-1)_{+}=\max \{|D u|-1,0\}
$$

so that

$$
D P=\chi_{\{|D u|>1\}} \cdot \frac{D u}{|D u|} \cdot D^{2} u .
$$




\section{$2.1 \quad$ Orlicz Spaces}

In this section we recall some basic properties of Orlicz spaces (for more details we refer to [1]).

Let $\Psi:[0, \infty) \rightarrow[0, \infty)$ be a Young function, that is $\Psi(0)=0, \Psi$ is increasing and convex. If $\Omega$ is a open subset of $\mathbb{R}^{n}$, we define the Orlicz space $L^{\Psi}(\Omega)$ generated by the Young function $\Psi$ as the set of the measurable functions $u: \Omega \rightarrow \mathbb{R}$ such that

$$
\int_{\Omega} \Psi\left(\frac{|u|}{\lambda}\right) d x<\infty
$$

for some $\lambda>0$. Once equipped with the Luxemburg norm

$$
\|u\|_{L^{\Psi}(\Omega)}=\inf \left\{\lambda>0: \int_{\Omega} \Psi\left(\frac{|u|}{\lambda}\right) d x \leqslant 1\right\}
$$

$L^{\Psi}(\Omega)$ is a Banach space. We define the space $W L^{\Psi}(\Omega)$ as the set

$$
W L^{\Psi}(\Omega)=\left\{u \in W^{1,1}(\Omega):|\nabla u| \in L^{\Psi}(\Omega)\right\} .
$$

The Zygmund space $L^{p} \log ^{\alpha} L(\Omega)$, for $1 \leqslant p<\infty, \alpha \in \mathbb{R}(\alpha \geqslant 0$ for $p=1)$, is defined as the Orlicz space $L^{\Psi}(\Omega)$ generated by the Young function

$$
\Psi(t) \simeq t^{p} \log ^{\alpha}(e+t) \quad \text { for every } t \geq t_{0} \geq 0 .
$$

Therefore, a measurable function $u$ on $\Omega$ belongs to $L^{p} \log ^{\alpha} L(\Omega)$ if

$$
\int_{\Omega}|u|^{p} \log ^{\alpha}(e+|u|) d x<\infty,
$$

and we recall that the quantity

$$
[u]_{p, \alpha}=\left[\int|u|^{p} \log ^{\alpha}\left(e+\frac{|u|}{\|u\|_{p}}\right) d x\right]^{\frac{1}{p}}
$$

is equivalent to the Luxemburg norm for $\alpha \geq 0$. For further needs, we record that the function $\Psi(t)=t^{p} \log ^{\alpha}(e+t), p>1, \alpha \in \mathbb{R}$, satisfy the so called $\Delta_{2}$ and $\nabla_{2}$ conditions. This is equivalent to the fact that

$$
\frac{\Psi(t)}{t^{p-\varepsilon}} \nearrow \quad \frac{\Psi(t)}{t^{p+\varepsilon}} \searrow \quad \text { for every } \varepsilon>0,
$$

and so, it can be easily checked that

$$
\Psi(\lambda t) \geq \lambda^{p+\varepsilon} \Psi(t) \quad \text { for every } \lambda \in(0,1)
$$

and

$$
\Psi(\mu t) \geq \mu^{p-\varepsilon} \Psi(t) \quad \text { for every } \mu>1
$$

From this and the definition of Luxenburg norm, we deduce that

$$
1=\int_{\Omega} \Psi\left(\frac{|f|}{\|f\|_{\Psi}}\right) d x \geq\left(\frac{1}{\|f\|_{\Psi}}\right)^{p+\varepsilon} \int_{\Omega} \Psi(|f|) \quad \text { if }\|f\|_{\Psi} \geq 1
$$

and

$$
1=\int_{\Omega} \Psi\left(\frac{|f|}{\|f\|_{\Psi}}\right) d x \geq\left(\frac{1}{\|f\|_{\Psi}}\right)^{p-\varepsilon} \int_{\Omega} \Psi(|f|) \quad \text { if }\|f\|_{\Psi} \leq 1
$$

We deduce that

$$
\int_{\Omega}|f|^{p} \log ^{\alpha}(e+|f|) d x \leq|| f \|_{\Psi}^{\vartheta}
$$

for some $\vartheta=\vartheta(p)>0$. 


\section{The a priori $L^{\infty}$ estimate}

This section is devoted to the proof of the a priori estimates that will be the crucial steps in the proofs of both Theorems 1 and 2 . The precise statement is the following one.

Theorem 4. Assume (F0)-(F4) hold, and let $f \in L^{n} \log ^{\alpha} L_{\mathrm{loc}}(\Omega)$ where $\alpha>3 n$ is the exponent appearing in (F4). Fix a ball $B_{r}\left(x_{0}\right) \Subset \Omega$, and functions $u, \bar{u} \in W^{1, p}\left(B_{r}\left(x_{0}\right) ; \mathbb{R}^{N}\right)$, and define

$$
\widetilde{\mathbb{F}}\left(v ; B_{r}\left(x_{0}\right)\right):=\mathbb{F}\left(v ; B_{r}\left(x_{0}\right)\right)+\int_{B_{r}\left(x_{0}\right)} \arctan \left(|v-\bar{u}(x)|^{2}\right) d x,
$$

where $\mathbb{F}$ is defined in 1.11). Let $v \in u+W_{0}^{1, p}\left(B_{r}\left(x_{0}\right) ; \mathbb{R}^{N}\right)$ be a minimizer of $\widetilde{\mathbb{F}}$, satisfying

$$
v \in W_{\mathrm{loc}}^{2,2}\left(B_{r}\left(x_{0}\right) ; \mathbb{R}^{N}\right) \cap W_{\mathrm{loc}}^{1, \infty}\left(B_{r}\left(x_{0}\right) ; \mathbb{R}^{N}\right) \quad \text { and } \quad|D v|^{p-2}\left|D^{2} v\right|^{2} \in L_{\mathrm{loc}}^{1}\left(B_{r}\left(x_{0}\right)\right) .
$$

Then, for every $B_{\bar{r}}(\bar{x}) \Subset B_{r}\left(x_{0}\right)$, every $0<\rho<r^{\prime} \leq \bar{r}$

$$
\sup _{B_{\rho}(\bar{x})}|D u| \leqslant C\left[\int_{B_{r^{\prime}}(\bar{x})}(1+|D u|)^{p} \mathrm{~d} x\right]^{\frac{1}{p}},
$$

for some constant $C=C\left(n, N, p, L_{1}, \nu, \rho, r^{\prime},\|k\|_{L^{n} \log ^{\alpha} L\left(B_{r^{\prime}}\right)},\|f\|_{L^{n} \log ^{\alpha} L\left(B_{r^{\prime}}\right)}\right)$. Moreover, one has

$$
\int_{B_{\rho}(\bar{x})} \frac{(|D u|-1)_{+}^{2}}{\left(1+(|D u|-1)_{+}\right)^{2}}|D u|^{p-2}\left|D^{2} u\right|^{2} d x \leq C \int_{B_{r^{\prime}}(\bar{x})}\left(1+|D u|^{p}\right) d x
$$

for some $C=C\left(n, N, p, L_{1}, \nu, \rho, r^{\prime},\|k\|_{L^{n} \log ^{\alpha} L\left(B_{r^{\prime}}\right)},\|f\|_{L^{n} \log ^{\alpha} L\left(B_{r^{\prime}}\right)}\right)$.

For the proof of the above result, the integral $\int_{B_{r}\left(x_{0}\right)} \arctan \left(|v-\bar{u}|^{2}\right) d x$ is a perturbation of $\mathbb{F}\left(v ; B_{r}\left(x_{0}\right)\right)$ that provides no difficulties. Indeed, denoting $g(x, v):=\arctan \left(|v-\bar{u}(x)|^{2}\right)$, we have that $g$ and its derivatives $g_{v^{\alpha}}, \alpha=1, \ldots, m$, are bounded. Thus, for the sake of clarity, we prefer to drop this perturbation term, and to state, and prove an a priori estimate for local minimizers of $\mathbb{F}(\cdot ; \Omega)$ only, as done in [10, see Theorem 5 below.

Theorem 5. Let $\mathcal{F}(x, \xi)$ satisfy assumptions $(\mathbf{F 0})-(\mathbf{F} 4)$, and let $f \in L^{n} \log ^{\alpha} L_{\mathrm{loc}}(\Omega)$ where $\alpha>$ $3 n$ is the exponent appearing in (F4). Assume that $u \in W_{\text {loc }}^{2,2}\left(\Omega, \mathbb{R}^{N}\right) \cap W_{\text {loc }}^{1, \infty}\left(\Omega, \mathbb{R}^{N}\right)$ is a local minimizer of the functional $\mathbb{F}(\cdot ; \Omega)$, and that $|D u|^{p-2}\left|D^{2} u\right|^{2} \in L_{\mathrm{loc}}^{1}(\Omega)$. Then the estimate

$$
\sup _{B_{\rho}}|D u| \leqslant \hat{C}\left[\int_{B_{R}}(1+|D u|)^{p} \mathrm{~d} x\right]^{\frac{1}{p}},
$$

holds for every concentric balls $B_{\rho} \subset B_{R} \Subset \Omega$. Moreover, the following second order Caccioppoli type inequality

$$
\int_{B_{\rho}} \frac{(|D u|-1)_{+}^{2}}{\left(1+(|D u|-1)_{+}\right)^{2}}|D u|^{p-2}\left|D^{2} u\right|^{2} d x \leq \hat{C} \int_{B_{2 R}}\left(1+|D u|^{p}\right) d x,
$$

holds for some $\hat{C}=\hat{C}\left(n, N, p, L_{1}, \nu, \rho, R,\|k\|_{L^{n} \log ^{\alpha} L\left(B_{2 R}\right)},\|f\|_{L^{n} \log ^{\alpha} L\left(B_{2 R}\right)}\right)$ and for every concentric balls $B_{\rho} \subset B_{R} \subset B_{2 R} \Subset \Omega$. 
Proof. We will prove the theorem in 3 steps.

Step 1. The first step is to prove that for every $\gamma \geqslant 0$ and for every non-negative function $\eta \in C_{0}^{\infty}(\Omega)$, there exists a positive constant $C=C\left(n, N, \nu, p, L_{1}\right)$ such that

$$
\begin{aligned}
\int_{\Omega} \eta^{2} \Phi(P) & |D u|^{p-2}\left|D^{2} u\right|^{2} d x \leq C(\gamma+1)^{2} \int_{\{x \in \Omega:|D u|>1\}} \eta^{2} k^{2}|D u|^{\gamma+p} d x \\
& +C \int_{\{x \in \Omega:|D u|>1\}}|D \eta|^{2}|D u|^{\gamma+p} d x+C(\gamma+1)^{2} \int_{\{x \in \Omega:|D u|>1\}} \eta^{2}|f|^{2}|D u|^{\gamma} d x
\end{aligned}
$$

where $\Phi$ is the function defined at (2.1) and $P=(|D u|-1)_{+}$.

Since $u$ is a local minimizer of $\mathbb{F}(\cdot ; \Omega)$, it satisfies the following integral identity

$$
\int_{\Omega}\left\langle D_{\xi} \mathcal{F}(x, D u), D \psi\right\rangle=\int_{\Omega} f \cdot \psi \quad \forall \psi \in C_{0}^{\infty}\left(\Omega, \mathbb{R}^{N}\right)
$$

By our assumptions on $u$ and a standard approximation argument, we can choose

$$
\psi \equiv \sum_{s} D_{x_{s}}\left(\eta^{2} \cdot \Phi(P) \cdot D_{x_{s}} u\right)
$$

where $\eta \in C_{0}^{\infty}(\Omega)$. Such a choice, together with an integration by parts in the left hand side of previous identity, yields

$$
-\sum_{s} \int_{\Omega}\left\langle D_{x_{s} \xi} \mathcal{F}(x, D u)+D_{\xi \xi} \mathcal{F}(x, D u) \cdot D_{x_{s}} D u, D\left(\eta^{2} \Phi(P) D_{x_{s}} u\right)\right\rangle=\sum_{s} \int_{\Omega} f \eta^{2} \Phi(P) D_{x_{s}} u
$$

We now use the product rule to calculate the derivatives of $\eta^{2} \cdot \Phi(P) \cdot D_{x_{s}} u$. This converts (3.6) into

$$
I_{1}+I_{2}+I_{3}+I_{4}+I_{5}+I_{6}+I_{7}+I_{8}+I_{9}=0
$$

where

$$
\begin{aligned}
& I_{1}=2 \sum_{s} \int_{\Omega}\left\langle D_{\xi \xi} \mathcal{F}(x, D u) \cdot D_{x_{s}} D u, D \eta \cdot D_{x_{s}} u\right\rangle \eta \Phi(P) d x, \\
& I_{2}=\sum_{s} \int_{\Omega}\left\langle D_{\xi \xi} \mathcal{F}(x, D u) \cdot D_{x_{s}} D u, D_{x_{s}} D u\right\rangle \eta^{2} \Phi(P) d x, \\
& I_{3}=\sum_{s} \int_{\Omega}\left\langle D_{\xi \xi} \mathcal{F}(x, D u) \cdot D_{x_{s}} D u, \Phi^{\prime}(P) D_{x_{s}} P \cdot D_{x_{s}} u\right\rangle \eta^{2} d x, \\
& I_{4}=2 \sum_{s} \int_{\Omega}\left\langle D_{x_{s} \xi} \mathcal{F}(x, D u), D \eta \cdot D_{x_{s}} u\right\rangle \eta \Phi(P) d x, \\
& I_{5}=\sum_{s} \int_{\Omega}\left\langle D_{x_{s} \xi} \mathcal{F}(x, D u), D_{x_{s}} D u\right\rangle \eta^{2} \Phi(P) d x, \\
& I_{6}=\sum_{s} \int_{\Omega}\left\langle D_{x_{s} \xi} \mathcal{F}(x, D u), \Phi^{\prime}(P) D_{x_{s}} P \cdot D_{x_{s}} u\right\rangle \eta^{2} d x, \\
& I_{7}=2 \sum_{s} \int_{\Omega} f \eta \Phi(P) D_{x_{s}} \eta \cdot D_{x_{s}} u d x, \\
& I_{8}=\sum_{s} \int_{\Omega} f \eta^{2} \Phi(P) D_{x_{s} x_{s}} u d x,
\end{aligned}
$$




$$
I_{9}=\sum_{s} \int_{\Omega} f \eta^{2} \Phi^{\prime}(P) D_{x_{s}} u \cdot D_{x_{s}} P d x .
$$

We will estimate each term separately. It is worth pointing out that the integrals $I_{i}$, with $i=1, \ldots, 6$ will be estimate with arguments similar to those in [13, 9. We will report here for the sake of completeness.

For the estimate of $I_{1}$, we use assumption (F3) and Young's inequality as follows

$$
\begin{aligned}
\left|I_{1}\right| & \leqslant 2 L_{1} \sum_{s} \int_{\Omega} \eta|D \eta||D u|^{p-2}\left|D_{x_{s}} D u\right|\left|D_{x_{s}} u\right| \Phi(P) d x \\
& \leqslant \varepsilon \int_{\Omega} \eta^{2}|D u|^{p-2}\left|D^{2} u\right|^{2} \Phi(P) d x+C_{\varepsilon}\left(L_{1}\right) \int_{\Omega}|D \eta|^{2}|D u|^{p} \Phi(P) d x,
\end{aligned}
$$

where $\varepsilon>0$ will be chosen later. In order to estimate $I_{4}$, we use assumption (F4) and Young's inequality as follows,

$$
\begin{aligned}
\left|I_{4}\right| & \leqslant 2 \int_{\Omega} \eta|D \eta| k|D u|^{p} \Phi(P) d x \\
& \leqslant C \int_{\Omega} \eta^{2} k^{2}|D u|^{p} \Phi(P) d x+C \int_{\Omega}|D \eta|^{2}|D u|^{p} \Phi(P) d x .
\end{aligned}
$$

We estimate $I_{5}$, using (F4) and Young's inequality again. Indeed

$$
\begin{aligned}
\left|I_{5}\right| & \leqslant \int_{\Omega} \eta^{2} k|D u|^{p-1}\left|D^{2} u\right| \Phi(P) d x \\
& \leqslant \varepsilon \int_{\Omega} \eta^{2}|D u|^{p-2}\left|D^{2} u\right|^{2} \Phi(P) d x+C_{\varepsilon} \int_{\Omega} \eta^{2} k^{2}|D u|^{p} \Phi(P) d x .
\end{aligned}
$$

For the estimate of $I_{6}$, again by virtue of assumption (F4), we have

$$
\begin{aligned}
\left|I_{6}\right| & \leqslant \sum_{s} \int_{\Omega} \eta^{2} k|D u|^{p-1}\left|D_{x_{s}} u\right| \Phi^{\prime}(P)\left|D_{x_{s}} P\right| d x \\
& \leqslant C \int_{\Omega} \eta^{2} k|D u|^{p} \Phi^{\prime}(P)|D P| d x \leqslant C \int_{\Omega} \eta^{2} k|D u|^{p} \Phi^{\prime}(P)\left|D^{2} u\right| \\
& =C \int_{\{|D u| \geq 2\}} \eta^{2} k|D u|^{p} \Phi^{\prime}(P)\left|D^{2} u\right|+C \int_{\{1<|D u|<2\}} \eta^{2} k|D u|^{p} \Phi^{\prime}(P)\left|D^{2} u\right|,
\end{aligned}
$$

where we used the equality in (2.4). Noting that

$$
|D u|=(|D u|-1)_{+}+1 \leq 2(|D u|-1)_{+} \quad \text { on the set }\{|D u| \geq 2\}
$$

and, recalling (2.2), we can estimate the first integral in the right hand side of previous inequality as follows

$$
\begin{aligned}
& C \int_{\{|D u| \geq 2\}} \eta^{2} k|D u|^{p} \Phi^{\prime}(P)\left|D^{2} u\right| \leq 2 C \int_{\{|D u| \geq 2\}} \eta^{2} k|D u|^{p-1} P \Phi^{\prime}(P)\left|D^{2} u\right| \\
& \leq 4 C(1+\gamma) \int_{\{|D u| \geq 2\}} \eta^{2} k|D u|^{p-1} \Phi(P)\left|D^{2} u\right| \\
& \leq \varepsilon \int_{\{|D u| \geq 2\}} \eta^{2}|D u|^{p-2} \Phi(P)\left|D^{2} u\right|^{2}+C_{\varepsilon}(1+\gamma)^{2} \int_{\{|D u| \geq 2\}} \eta^{2} k^{2}|D u|^{p} \Phi(P) .
\end{aligned}
$$


After setting $\Gamma_{\gamma}=2(1+\gamma)>0$, we multiply and divide the last integrand in (3.10) by $\left(\frac{\delta+P}{\Gamma_{\gamma}}\right)^{1 / 2}$ with $0<\delta<1$, and use Young's inequality, thus obtaining

$$
\begin{aligned}
& C \int_{\{1<|D u|<2\}} \eta^{2} \Phi^{\prime}(P)\left\{\frac{\delta+P}{\Gamma_{\gamma}}|D u|^{p-2}\left|D^{2} u\right|^{2}\right\}^{\frac{1}{2}} \times\left\{\frac{\Gamma_{\gamma}}{\delta+P} k^{2}|D u|^{p+2}\right\}^{\frac{1}{2}} d x \\
\leqslant & \varepsilon \int_{\{1<|D u|<2\}} \eta^{2} \Phi^{\prime}(P) \frac{\delta+P}{\Gamma_{\gamma}}|D u|^{p-2}\left|D^{2} u\right|^{2} d x \\
& +C_{\varepsilon} \int_{\{1<|D u|<2\}} \eta^{2} k^{2} \Phi^{\prime}(P) \frac{\Gamma_{\gamma}}{\delta+P}|D u|^{p+2} d x \\
\leq & \frac{\varepsilon}{\Gamma_{\gamma}} \int_{\{1<|D u|<2\}} \eta^{2} \Phi^{\prime}(P) P|D u|^{p-2}\left|D^{2} u\right|^{2} d x+\frac{\varepsilon \delta}{\Gamma_{\gamma}} \int_{\{1<|D u|<2\}} \eta^{2} \Phi^{\prime}(P)|D u|^{p-2}\left|D^{2} u\right|^{2} d x \\
& +C_{\varepsilon} \int_{\{1<|D u|<2\}} \eta^{2} k^{2} \Phi^{\prime}(P) \frac{\Gamma_{\gamma}}{\delta+P}|D u|^{p+2} d x \\
\leqslant & \varepsilon \int_{\{1<|D u|<2\}} \eta^{2} \Phi(P)|D u|^{p-2}\left|D^{2} u\right|^{2} d x+\varepsilon \delta \tilde{C}_{\gamma} \int_{\{1<|D u|<2\}} \eta^{2}\left|D^{2} u\right|^{2}|D u|^{p-2} d x \\
& +\Gamma_{\gamma}^{2} C_{\varepsilon} \int_{\{1<|D u|<2\}} \eta^{2} k^{2}|D u|^{\gamma+p} d x
\end{aligned}
$$

where, in the last line we used (2.2), (2.3) and the fact that, since $\frac{P}{\delta+P} \leqslant 1$, we have

$$
\begin{aligned}
(\delta+P)^{-1} \Phi^{\prime}(P) & =\Phi^{\prime}(P) \cdot \frac{P}{\delta+P} \cdot P^{-1} \\
& \leqslant \Gamma_{\gamma} \Phi(P) \cdot P^{-2}=\Gamma_{\gamma}(1+P)^{\gamma-2} \\
& =\Gamma_{\gamma}|D u|^{\gamma-2} \quad \text { in the set } \quad\{1<|D u|<2\} .
\end{aligned}
$$

Plugging (3.12) and (3.13) into (3.10), we get

$$
\begin{aligned}
\left|I_{6}\right| & \leqslant 2 \varepsilon \int_{\Omega} \eta^{2} \Phi(P)|D u|^{p-2}\left|D^{2} u\right|^{2} d x+\varepsilon \delta \tilde{C}_{\gamma} \int_{\Omega} \eta^{2}\left|D^{2} u\right|^{2}|D u|^{p-2} d x \\
& +\Gamma_{\gamma}^{2} C_{\varepsilon} \int_{\{|D u|>1\}} \eta^{2} k^{2}|D u|^{\gamma+p} d x .
\end{aligned}
$$

By virtue of the assumption $\left|D^{2} u\right|^{2}|D u|^{p-2} \in L_{\text {loc }}^{1}(\Omega)$, we can let $\delta \rightarrow 0$ in previous estimate thus getting

$$
\left|I_{6}\right| \leq 2 \varepsilon \int_{\Omega} \eta^{2} \Phi(P)|D u|^{p-2}\left|D^{2} u\right|^{2} d x+C_{\varepsilon}(1+\gamma)^{2} \int_{\{|D u|>1\}} \eta^{2} k^{2}|D u|^{\gamma+p} d x,
$$

where we used that $\Gamma_{\gamma} \sim(\gamma+1)$. For $I_{7}$, using Young's inequality and recalling that $P=(|D u|-1)_{+}$, we get

$$
\begin{aligned}
\left|I_{7}\right| & \leq 2 \int_{\Omega} \eta|D \eta||f||D u| \Phi(P)=2 \int_{\{|D u|>1\}} \eta|D \eta||f||D u|^{\gamma-1}(|D u|-1)^{2} \\
& \leq C \int_{\{|D u|>1\}} \eta^{2}|f|^{2}(|D u|-1)^{2}|D u|^{\gamma-p}+C \int_{\{|D u|>1\}}|D \eta|^{2}(|D u|-1)^{2}|D u|^{\gamma+p-2} \\
& =C \int_{\{|D u|>1\}} \eta^{2}|f|^{2} \Phi(P)|D u|^{2-p}+C \int_{\{|D u|>1\}}|D \eta|^{2} \Phi(P)|D u|^{p} \\
& \leq C \int_{\{|D u|>1\}} \eta^{2}|f|^{2} \Phi(P)+C \int_{\{|D u|>1\}}|D \eta|^{2} \Phi(P)|D u|^{p},
\end{aligned}
$$


where we used that $p \geq 2$ and that the set of integration is $\{|D u|>1\}$. Concerning $I_{8}$ and $I_{9}$, by (3.11) and Young's inequality, we have

$$
\begin{aligned}
\left|I_{8}\right|+\left|I_{9}\right| & \leq \int_{\Omega} \eta^{2}|f|\left|D^{2} u\right| \Phi(P)+\int_{\Omega} \eta^{2}|f||D u|\left|D^{2} u\right| \Phi^{\prime}(P) \\
& =\int_{\{|D u|>1\}} \eta^{2}|f|\left|D^{2} u\right| \Phi(P)+\int_{\{|D u| \geq 2\}} \eta^{2}|f||D u|\left|D^{2} u\right| \Phi^{\prime}(P)+\int_{\{1<|D u|<2\}} \eta^{2}|f||D u|\left|D^{2} u\right| \Phi^{\prime}(P) \\
& \leq \int_{\{|D u|>1\}} \eta^{2}|f|\left|D^{2} u\right| \Phi(P)+2 \int_{\{|D u| \geq 2\}} \eta^{2}|f|\left|D^{2} u\right| P \Phi^{\prime}(P)+\int_{\{1<|D u|<2\}} \eta^{2}|f||D u|\left|D^{2} u\right| \Phi^{\prime}(P) \\
& \leq c \Gamma_{\gamma} \int_{\{|D u|>1\}} \eta^{2}|f|\left|D^{2} u\right| \Phi(P)+\int_{\{1<|D u|<2\}} \eta^{2}|f||D u|\left|D^{2} u\right| \Phi^{\prime}(P) .
\end{aligned}
$$

The first integral in the right hand side of (3.17) can be estimated by Young's inequality as follows

$$
\begin{aligned}
& c \Gamma_{\gamma} \int_{\{|D u|>1\}} \eta^{2}|f|\left|D^{2} u\right| \Phi(P) \\
& \leq \varepsilon \int_{\{|D u|>1\}} \eta^{2}\left|D^{2} u\right|^{2} \Phi(P)+C_{\varepsilon} \cdot \Gamma_{\gamma}^{2} \int_{\{|D u|>1\}} \eta^{2}|f|^{2} \Phi(P) \\
& \leq \varepsilon \int_{\Omega} \eta^{2}|D u|^{p-2}\left|D^{2} u\right|^{2} \Phi(P)+C_{\varepsilon} \cdot \Gamma_{\gamma}^{2} \int_{\Omega} \eta^{2}|f|^{2} \Phi(P),
\end{aligned}
$$

where we used that $|D u|^{p-2}>1$ on the set $|D u|>1$ since $p>2$, and that, as before, $\Gamma_{\gamma} \sim(\gamma+1)$. To estimate the second integral in (3.17), we argue as we did for $I_{6}$, multiplying and dividing it by $\left(\frac{\delta+P}{\Gamma_{\gamma}}\right)^{\frac{1}{2}}$ with $0<\delta<1$ and using Young's inequality. We get

$$
\begin{aligned}
& \int_{\{1<|D u|<2\}} \eta^{2}|f||D u|\left|D^{2} u\right| \Phi^{\prime}(P)=\int_{\{1<|D u|<2\}} \eta^{2} \Phi^{\prime}(P)\left\{\frac{\delta+P}{\Gamma_{\gamma}}\left|D^{2} u\right|^{2}\right\}^{\frac{1}{2}}\left\{|f|^{2}|D u|^{2} \frac{\Gamma_{\gamma}}{\delta+P}\right\}^{\frac{1}{2}} \\
\leq & \frac{\varepsilon}{\Gamma_{\gamma}} \int_{\{1<|D u|<2\}} \eta^{2}(\delta+P)\left|D^{2} u\right|^{2} \Phi^{\prime}(P)+C_{\varepsilon} \cdot \Gamma_{\gamma} \int_{\{1<|D u|<2\}} \eta^{2} \frac{|D u|^{2}|f|^{2}}{(\delta+P)} \Phi^{\prime}(P) \\
\leq & \frac{\varepsilon}{\Gamma_{\gamma}} \int_{\{1<|D u|<2\}} \eta^{2}\left|D^{2} u\right|^{2} P \Phi^{\prime}(P)+\frac{\varepsilon}{\Gamma_{\gamma}} \delta \int_{\{1<|D u|<2\}} \eta^{2}\left|D^{2} u\right|^{2} \Phi^{\prime}(P) \\
& +C_{\varepsilon} \cdot \Gamma_{\gamma} \int_{\{1<|D u|<2\}} \eta^{2} \frac{|D u|^{2}|f|^{2}}{(\delta+P)} \Phi^{\prime}(P) \\
\leq & \int_{\{1<|D u|<2\}} \eta^{2}\left|D^{2} u\right|^{2} \Phi(P)+\delta \varepsilon \tilde{C}_{\gamma} \int_{\{1<|D u|<2\}} \eta^{2}\left|D^{2} u\right|^{2}|D u|^{p-2} \\
& +C_{\varepsilon} \Gamma_{\gamma}^{2} \int_{\{1<|D u|<2\}} \eta^{2}|D u|^{\gamma}|f|^{2} \\
\leq & \int_{\{1<|D u|<2\}} \eta^{2}\left|D^{2} u\right|^{2}|D u|^{p-2} \Phi(P)+\delta \varepsilon \tilde{C}_{\gamma} \int_{\{1<|D u|<2\}} \eta^{2}\left|D^{2} u\right|^{2}|D u|^{p-2} \\
& +C_{\varepsilon} \Gamma_{\gamma}^{2} \int_{\{1<|D u|<2\}} \eta^{2}|D u|^{\gamma}|f|^{2},
\end{aligned}
$$

where we used that $\delta+P \leq 2 P$ in the set $\{|D u|>2\}$, inequalities (2.2), (2.3), (3.14) and that $|D u|^{p-2}>1$ in the set $\{|D u|>1\}$. Inserting (3.18) and (3.19) in (3.17) and letting $\delta \rightarrow 0$, we get

$$
\left|I_{8}\right|+\left|I_{9}\right| \leq 2 \varepsilon \int_{\Omega} \eta^{2}|D u|^{p-2}\left|D^{2} u\right|^{2} \Phi(P)+C_{\varepsilon} \Gamma_{\gamma}^{2} \int_{\{|D u|>1\}} \eta^{2}|D u|^{\gamma}|f|^{2} .
$$


We remind that

$$
I_{2}+I_{3}=-I_{1}-I_{4}-I_{5}-I_{6}-I_{7}-I_{8}-I_{9}
$$

We now elaborate on the precise form of $D_{\xi \xi} \mathcal{F}(x, \xi)$ to estimate $I_{3}$. To do this, we abuse of notation and for every scalar $t$ we denote $F^{\prime}(x, t)=\partial_{t} F(x, t)$ and $F^{\prime \prime}(x, t)=\partial_{t t} F(x, t)$. By (F1), for every $\xi \in \mathbb{R}^{n \times N} \backslash\{0\}$ one has

$$
D_{\xi \xi} \mathcal{F}(x, \xi)=\left(\frac{F^{\prime \prime}(x,|\xi|)}{|\xi|^{2}}-\frac{F^{\prime}(x,|\xi|)}{|\xi|^{3}}\right) \xi \otimes \xi+\frac{F^{\prime}(x,|\xi|)}{|\xi|} \mathbf{I d}_{\mathbb{R}^{n \times N}} .
$$

Componentwise,

$$
\begin{aligned}
D_{\xi_{j}^{\beta} \xi_{i}^{\alpha}} \mathcal{F}(x, \xi) & =D_{\xi_{j}^{\beta}}\left(F^{\prime}(x,|\xi|) \frac{\xi_{i}^{\alpha}}{|\xi|}\right) \\
& =\left(\frac{F^{\prime \prime}(x,|\xi|)}{|\xi|^{2}}-\frac{F^{\prime}(x,|\xi|)}{|\xi|^{3}}\right) \xi_{i}^{\alpha} \xi_{j}^{\beta}+\frac{F^{\prime}(x,|\xi|)}{|\xi|} \delta_{\xi_{i}^{\alpha} \xi_{j}^{\beta} .}
\end{aligned}
$$

Recalling the equality in (2.4), it is well known that for a.e. $x \in\{|D u| \geq 1\}$, we have

$$
\begin{gathered}
\sum_{s}\left\langle D_{\xi \xi} \mathcal{F}(x, D u) \cdot D_{x_{s}} D u, D P \cdot D_{x_{s}} u\right\rangle=\sum_{s, i, j, \alpha, \beta} D_{\xi_{j}^{\beta} \xi_{i}^{\alpha}} \mathcal{F}(x, D u) u_{x_{s}}^{\alpha} u_{x_{s} x_{j}}^{\beta} P_{x_{i}} \\
=\left(\frac{F^{\prime \prime}(x,|D u|)}{|D u|}-\frac{F^{\prime}(x,|D u|)}{|D u|^{2}}\right) \sum_{\alpha}\left(\sum_{i} u_{x_{i}}^{\alpha}(|D u|)_{x_{i}}\right)^{2}+F^{\prime}(x,|D u|)|D(|D u|)|^{2} .
\end{gathered}
$$

Thus,

$$
\begin{aligned}
I_{3} & =\int_{\Omega} \eta^{2} \Phi^{\prime}(P) \frac{F^{\prime \prime}(x,|D u|)}{|D u|} \sum_{\alpha}\left(\sum_{i} u_{x_{i}}^{\alpha}(|D u|)_{x_{i}}\right)^{2} d x \\
& +\int_{\Omega} \eta^{2} \Phi^{\prime}(P) F^{\prime}(x,|D u|)\left(|D(|D u|)|^{2}-\frac{\sum_{\alpha}\left(\sum_{i} u_{x_{i}}^{\alpha}(|D u|)_{x_{i}}\right)^{2}}{|D u|^{2}}\right) d x .
\end{aligned}
$$

The use of the Cauchy-Schwartz inequality implies

$$
\sum_{\alpha}\left(\sum_{i} u_{x_{i}}^{\alpha}(|D u|)_{x_{i}}\right)^{2} \leq|D u|^{2}|D(|D u|)|^{2} .
$$

and since

$$
\Phi^{\prime}(t)=(1+t)^{\gamma-3} t(\gamma t+2)
$$

is nonnegative for every $t \geq 0$ and, by (F2), $F^{\prime}(x,|D u|) \geq 0$, then we conclude that

$$
I_{3} \geq \int_{\Omega} \eta^{2} \Phi^{\prime}(P) \frac{F^{\prime \prime}(x,|D u|)}{|D u|} \sum_{\alpha}\left(\sum_{i} u_{x_{i}}^{\alpha}(|D u|)_{x_{i}}\right)^{2} d x \geq 0 .
$$

Therefore, using that $I_{3} \geqslant 0$ together with (3.21) we have

$$
I_{2} \leqslant\left|I_{1}\right|+\left|I_{4}\right|+\left|I_{5}\right|+\left|I_{6}\right|+\left|I_{7}\right|+\left|I_{8}\right|+\left|I_{9}\right| .
$$

On the other hand, the ellipticity assumption (F2) gives that

$$
I_{2} \geqslant \nu \int_{\Omega} \eta^{2} \cdot \Phi(P) \cdot|D u|^{p-2} \cdot\left|D^{2} u\right|^{2} d x .
$$


Inserting estimates (3.7), (3.8), (3.9), 3.15), 3.16), (3.20) and (3.26) into (3.25), we obtain

$$
\begin{aligned}
& \nu \int_{\Omega} \eta^{2} \cdot \Phi(P)|D u|^{p-2}\left|D^{2} u\right|^{2} d x \\
& \leq 6 \varepsilon \int_{\Omega} \eta^{2} \Phi(P)|D u|^{p-2}\left|D^{2} u\right|^{2} d x+\left.C_{\varepsilon}\left(L_{1}\right) \int_{\{|D u|>1\}}|D \eta|^{2}|| D u\right|^{p+\gamma} d x \\
& +C_{\varepsilon} \int_{\{|D u|>1\}} \eta^{2} k^{2}|D u|^{p+\gamma} d x+C_{\epsilon}(\gamma+1)^{2} \int_{\{|D u|>1\}} \eta^{2} k^{2}|D u|^{p+\gamma} d x \\
& +C_{\varepsilon}(\gamma+1)^{2} \int_{\{|D u|>1\}} \eta^{2}|f|^{2}|D u|^{\gamma} d x
\end{aligned}
$$

where we used that $\Phi(P) \leq(1+P)^{\gamma}$. We now choose $\varepsilon=\frac{\nu}{12}$, and reabsorb the first integral in the right hand side by the left hand side. We obtain

$$
\begin{aligned}
& \int_{\Omega} \eta^{2} \cdot \Phi(P)|D u|^{p-2}\left|D^{2} u\right|^{2} d x \\
& \leq\left. C \int_{\{|D u|>1\}}|D \eta|^{2}|| D u\right|^{p+\gamma} d x+C \int_{\{|D u|>1\}} \eta^{2} k^{2}|D u|^{p+\gamma} d x \\
& +C(\gamma+1)^{2} \int_{\{|D u|>1\}} \eta^{2} k^{2}|D u|^{p+\gamma} d x+C(\gamma+1)^{2} \int_{\{|D u|>1\}} \eta^{2}|f|^{2}|D u|^{\gamma} d x \\
& \leq\left. C \int_{\{|D u|>1\}}|D \eta|^{2}|| D u\right|^{p+\gamma} d x+C(\gamma+1)^{2} \int_{\{|D u|>1\}} \eta^{2} k^{2}|D u|^{p+\gamma} d x \\
& +C(\gamma+1)^{2} \int_{\{|D u|>1\}} \eta^{2}|f|^{2}|D u|^{\gamma} d x
\end{aligned}
$$

with $C=C\left(n, N, p, \nu, L_{1}\right)$, that is inequality (3.5).

Step 2. Fix a ball $B_{R}\left(x_{0}\right) \Subset \Omega$ and radii $0<\rho<r<t<R$. Let $\eta \in C_{0}^{\infty}\left(B_{t}\right)$ be a cut off function such that $\eta \equiv 1$ on $B_{r}$ and $|D \eta| \leqslant \frac{C}{t-r}$. Recalling the definition of $\Phi(t)$ and that $|D u|=1+P$ on the set $\{|D u|>1\}$, inequality (3.5) can be written as follows

$$
\begin{aligned}
& \int_{\{|D u|>1\}} \eta^{2} P^{2}(1+P)^{\gamma+p-4}\left|D^{2} u\right|^{2} d x \\
\leq & C \int_{\{|D u|>1\}}|D \eta|^{2}(1+P)^{\gamma+p} d x+C(\gamma+1)^{2} \int_{\{|D u|>1\}} \eta^{2}\left(k^{2}+|f|^{2}\right)(1+P)^{\gamma+p} d x \\
= & : J_{1}+J_{2}
\end{aligned}
$$

Using the properties of $\eta$, we get

$$
J_{1} \leqslant \frac{C}{(t-r)^{2}} \int_{B_{t} \cap\{|D u|>1\}}(1+P)^{(\gamma+p)} d x .
$$

In order to estimate $J_{2}$, it is convenient to split the ball $B_{t}$ as follows

$$
B_{t}=\left\{x \in B_{t}: k^{2}+|f|^{2} \leq \exp \left(\frac{1}{\varepsilon}\right)-e\right\} \bigcup\left\{x \in B_{t}: k^{2}+|f|^{2}>\exp \left(\frac{1}{\varepsilon}\right)-e\right\}=: B_{\varepsilon}^{1} \cup B_{\varepsilon}^{2}
$$

so that

$$
J_{2}=C(\gamma+1)^{2}\left[\int_{B_{\varepsilon}^{1} \cap\{|D u|>1\}}\left(k^{2}+|f|^{2}\right)(1+P)^{\gamma+p} d x+\int_{B_{\varepsilon}^{2} \cap\{|D u|>1\}}\left(k^{2}+|f|^{2}\right)(1+P)^{\gamma+p} d x\right]
$$




$$
\begin{aligned}
& \leq C(\gamma+1)^{2}\left[\left(\exp \left(\frac{1}{\varepsilon}\right)-e\right) \int_{B_{t} \cap\{|D u|>1\}}(1+P)^{\gamma+p} d x+\int_{B_{\varepsilon}^{2} \cap\{|D u|>1\}}\left(k^{2}+|f|^{2}\right)(1+P)^{\gamma+p} d x\right] \\
& \leq C(\gamma+1)^{2}\left[\exp \left(\frac{1}{\varepsilon}\right) \int_{B_{t} \cap\{|D u|>1\}}(1+P)^{\gamma+p} d x+\int_{B_{\varepsilon}^{2} \cap\{|D u|>1\}}\left(k^{2}+|f|^{2}\right)(1+P)^{\gamma+p} d x\right] \\
& =: J_{2}^{1}+J_{2}^{2}
\end{aligned}
$$

We estimate $J_{2}^{2}$, by virtue of the assumptions $k, f \in L^{n} \log ^{\alpha} L_{\mathrm{loc}}(\Omega)$. In fact, we have

$$
\begin{aligned}
J_{2}^{2}=C(\gamma+1)^{2} & \int_{B_{\varepsilon}^{2} \cap\{|D u|>1\}}\left(k^{2}+|f|^{2}\right) \log ^{\frac{2 \alpha}{n}}\left(e+k^{2}+|f|^{2}\right) \log ^{-\frac{2 \alpha}{n}}\left(e+k^{2}+|f|^{2}\right)(1+P)^{\gamma+p} d x \\
\leq C(\gamma+1)^{2} & \left(\int_{B_{\varepsilon}^{2} \cap\{|D u|>1\}}\left(k^{n}+|f|^{n}\right) \log ^{\alpha}\left(e+k^{2}+|f|^{2}\right) d x\right)^{\frac{2}{n}} \\
& \cdot\left(\int_{B_{\varepsilon}^{2} \cap\{|D u|>1\}} \log ^{-\frac{2 \alpha}{n-2}}\left(e+k^{2}+|f|^{2}\right)(1+P)^{\frac{n(\gamma+p)}{n-2}} d x\right)^{\frac{n-2}{n}} \\
\leq C \varepsilon^{\frac{2 \alpha}{n}}(\gamma+1)^{2}|| k+|f| \|_{L^{n}}^{\vartheta} \log ^{\alpha} L & \left(\int_{B_{\varepsilon}^{2} \cap\{|D u|>1\}}(1+P)^{\frac{n(\gamma+p)}{n-2}} d x\right)^{\frac{n-2}{n}}
\end{aligned}
$$

where we used Hölder's inequality, the definition of $B_{\varepsilon}^{2}(2.6)$, and where now $\vartheta=\vartheta(n)$. Inserting (3.31) in (3.30), we obtain

$$
\begin{aligned}
J_{2} \leq C(\gamma & +1)^{2} \exp \left(\frac{1}{\varepsilon}\right) \int_{B_{t} \cap\{|D u|>1\}}(1+P)^{\gamma+p} d x \\
& +C \varepsilon^{\frac{2 \alpha}{n}}(\gamma+1)^{2}|| k+|f| \|_{L^{n} \log ^{\alpha} L}^{\vartheta}\left(\int_{B_{\varepsilon}^{2} \cap\{|D u|>1\}}(1+P)^{\frac{n(\gamma+p)}{n-2}} d x\right)^{\frac{n-2}{n}}
\end{aligned}
$$

Setting

$$
E_{R}:=\|k\|_{L^{n} \log ^{\alpha} L\left(B_{R}\right)}^{\vartheta}+\|f\|_{L^{n} \log ^{\alpha} L\left(B_{R}\right)}^{\vartheta}
$$

and inserting (3.29) and (3.32) into (3.28), and using the notation in (3.33), we get

$$
\begin{aligned}
& \int_{\{|D u|>1\}} \eta^{2} P^{2}(1+P)^{\gamma+p-4}\left|D^{2} u\right|^{2} d x \leq \frac{C}{(t-r)^{2}} \int_{B_{t}}(1+P)^{(\gamma+p)} d x \\
& \quad+C(\gamma+1)^{2} \exp \left(\frac{1}{\varepsilon}\right) \int_{B_{t}}(1+P)^{\gamma+p} d x \\
& \quad+C \varepsilon^{\frac{2 \alpha}{n}}(\gamma+1)^{2} E_{R}\left(\int_{B_{t}}(1+P)^{\frac{n(\gamma+p)}{n-2}} d x\right)^{\frac{n-2}{n}} .
\end{aligned}
$$

Following [13], for $t \geq 0$ we consider the auxiliary function

$$
G(t)=1+\int_{0}^{t}(1+s)^{\frac{\gamma+p-4}{2}} s d s
$$

and, recall that, by Lemma 2.2 in [15], the following inequalities

$$
\frac{1}{2(\gamma+p)^{2}}(1+t)^{\frac{\gamma+p}{2}} \leq G(t) \leq 2(1+t)^{\frac{\gamma+p}{2}}, \quad G^{\prime}(t)=t(1+t)^{\frac{\gamma+p-4}{2}}
$$


hold true. Let us denote by

$$
2^{*}=\left\{\begin{array}{l}
\frac{2 n}{n-2} \quad \text { if } n>2 \\
\text { any exponent } \quad \text { if } n=2 .
\end{array}\right.
$$

We consider now the case $n>2$. By the Sobolev imbedding Theorem, we get

$$
\left(\int_{\Omega}|\eta G(P)|^{2^{*}} d x\right)^{\frac{2}{2^{*}}} \leq C \int_{\Omega}|D(\eta G(P))|^{2} d x \leq C \int_{\Omega}|D \eta|^{2} G(P)^{2} d x+C \int_{\Omega} \eta^{2} G^{\prime}(P)^{2}|D P|^{2} d x
$$

Using the properties of $G(t)$ at (3.35) in the previous inequality we obtain

$$
\begin{aligned}
\frac{1}{(\gamma+p)^{4}} & \left(\int_{\Omega} \eta^{2^{*}}(1+P)^{\frac{2^{*}(\gamma+p)}{2}} d x\right)^{\frac{2}{2^{*}}} \\
& \leq c \int_{\Omega}|D \eta|^{2}(1+P)^{\gamma+p} d x+c \int_{\Omega} \eta^{2}(1+P)^{\gamma+p-4} P^{2}|D P|^{2} d x \\
& \leq \frac{c}{(t-r)^{2}} \int_{B_{t}}(1+P)^{\gamma+p} d x+c \int_{\Omega} \eta^{2} \Phi(P)(1+P)^{p-2}\left|D^{2} u\right|^{2} d x
\end{aligned}
$$

where we also used the properties of the function $\eta$. Inserting estimate (3.36) in (3.34) and using that $\eta \equiv 1$ on $B_{r}$, we get

$$
\begin{gathered}
\left(\int_{B_{r}}(1+P)^{\left.\frac{n(\gamma+p)}{n-2} d x\right)^{\frac{n-2}{n}} \leq} \frac{C(\gamma+p)^{4}}{(t-r)^{2}} \int_{B_{t}}(1+P)^{(\gamma+p)} d x+C(\gamma+p)^{6} \exp \left(\frac{1}{\varepsilon}\right) \int_{B_{t}}(1+P)^{\gamma+p} d x\right. \\
\quad+C \varepsilon^{\frac{2 \alpha}{n}}(\gamma+p)^{6} E_{R}^{2}\left(\int_{B_{t}}(1+P)^{\frac{n(\gamma+p)}{n-2}} d x\right)^{\frac{n-2}{n}}
\end{gathered}
$$

with $C=C\left(n, N, p, \nu, L_{1}\right)$. Choosing $\varepsilon$ such that

$$
C(\gamma+p)^{6} E_{R} \cdot \varepsilon^{\frac{2 \alpha}{n}}=\frac{1}{2} \quad \Longleftrightarrow \quad \frac{1}{\varepsilon}=\left(2 C E_{R}\right)^{\frac{n}{2 \alpha}}(\gamma+p)^{\frac{3 n}{\alpha}}
$$

and setting $\Theta=2 C E_{R}$, we get

$$
\begin{aligned}
\left(\int_{B_{r}}(1+P)^{\frac{n(\gamma+p)}{n-2}} d x\right)^{\frac{n-2}{n}} & \leq \frac{1}{2}\left(\int_{B_{t}}(1+P)^{\frac{n(\gamma+p)}{n-2}} d x\right)^{\frac{n-2}{n}}+\frac{C(\gamma+p)^{4}}{(t-r)^{2}} \int_{B_{R}}(1+P)^{(\gamma+p)} d x \\
& +C(\gamma+p)^{6} \cdot \exp \left(\Theta^{\frac{n}{2 \alpha}}(\gamma+p)^{\frac{3 n}{\alpha}}\right) \int_{B_{R}}(1+P)^{(\gamma+p)} d x
\end{aligned}
$$

By the iteration Lemma 3 we infer that

$$
\begin{gathered}
\left(\int_{B_{\rho}}(1+P)^{\frac{n(\gamma+p)}{n-2}} d x\right)^{\frac{n-2}{n}} \leq \frac{C(\gamma+p)^{4}}{(R-\rho)^{2}} \int_{B_{R}}(1+P)^{(\gamma+p)} d x \\
+C(\gamma+p)^{6} \cdot \exp \left(\Theta^{\frac{n}{2 \alpha}}(\gamma+p)^{\frac{3 n}{\alpha}}\right) \int_{B_{R}}(1+P)^{(\gamma+p)} d x \\
\leq C \frac{(\gamma+p)^{6} \cdot \exp \left(\Theta^{\frac{n}{2 \alpha}}(\gamma+p)^{\frac{3 n}{\alpha}}\right)}{(R-\rho)^{2}} \int_{B_{R}}(1+P)^{(\gamma+p)} d x,
\end{gathered}
$$

since we may suppose without loss of generality that $0<R-\rho<1$.

Step 3. Let us define the decreasing sequence of radii $\rho_{j}, j \in \mathbb{N}$, by setting

$$
\rho_{j}:=\rho+\frac{R-\rho}{2^{j}}
$$


and the increasing sequence of exponents

$$
p_{j}=p\left(\frac{2^{*}}{2}\right)^{j}
$$

Since $\gamma \geq 0$ can take any value, estimate (3.37) can be written on every ball $B_{\rho_{j}}$ as follows

$$
\left(\int_{B_{\rho_{j+1}}}(1+P)^{p_{j+1}} d x\right)^{\frac{1}{p_{j+1}}} \leqslant \frac{C^{\frac{1}{p_{j}}} p_{j}^{\frac{6}{p_{j}}}\left(\exp \left(\Theta \frac{n}{2 \alpha} p_{j}^{\frac{3 n}{\alpha}}\right)\right)^{\frac{1}{p_{j}}}}{\left(\rho_{j}-\rho_{j+1}\right)^{\frac{2}{p_{j}}}}\left(\int_{B_{\rho_{j}}}(1+P)^{p_{j}} d x\right)^{\frac{1}{p_{j}}}
$$

Iterating estimate 3.38 we obtain

$$
\begin{aligned}
\left(\int_{B_{\rho}}(1+P)^{p_{m+1}} d x\right)^{\frac{1}{p_{m+1}}} & \leqslant \prod_{j=0}^{m} \frac{C^{\frac{1}{p_{j}}} p_{j}^{\frac{6}{p_{j}}}\left(\exp \left(\Theta^{\frac{n}{2 \alpha}} p_{j}^{\frac{3 n}{\alpha}}\right)\right)^{\frac{1}{p_{j}}}}{\left(\rho_{j}-\rho_{j+1}\right)^{\frac{2}{p_{j}}}}\left(\int_{B_{R}}(1+P)^{p} d x\right)^{\frac{1}{p}} \\
& \leqslant \prod_{j=0}^{m} \frac{C^{\frac{1}{p_{j}}} 4^{\frac{j+1}{p_{j}}} p_{j}^{\frac{6}{p_{j}}}\left(\exp \left(\Theta^{\frac{n}{2 \alpha}} p_{j}^{\frac{3 n}{\alpha}}\right)\right)^{\frac{1}{p_{j}}}}{(R-\rho)^{\frac{2}{p_{j}}}}\left(\int_{B_{R}}(1+P)^{p} d x\right)^{\frac{1}{p}}
\end{aligned}
$$

where we used the definition of $\rho_{j}$. Now, we want to prove that the product in the right hand side of previous estimate is bounded by a constant. To this aim we write

$$
\begin{aligned}
& \prod_{j=0}^{m} \frac{C^{\frac{1}{p_{j}}} 4^{\frac{j+1}{p_{j}}} p_{j}^{\frac{6}{p_{j}}}\left(\exp \left(\Theta^{\frac{n}{2 \alpha}} p_{j}^{\frac{3 n}{\alpha}}\right)\right)^{\frac{1}{p_{j}}}}{(R-\rho)^{\frac{2}{p_{j}}}}=\prod_{j=0}^{m} \frac{C^{\frac{1}{p_{j}}} 4^{\frac{j+1}{p_{j}}} p_{j}^{\frac{6}{p_{j}}}}{(R-\rho)^{\frac{2}{p_{j}}}} \prod_{j=0}^{m} \exp \left(\Theta^{\frac{n}{2 \alpha}} p_{j}^{\frac{3 n}{\alpha}-1}\right) \\
= & \prod_{j=0}^{m} \frac{C^{\frac{1}{p_{j}}} 4^{\frac{j+1}{p_{j}}} p_{j}^{\frac{6}{p_{j}}}}{(R-\rho)^{\frac{2}{p_{j}}}} \exp \left(\Theta^{\frac{n}{2 \alpha}} \sum_{j=0}^{m} p_{j}^{\frac{3 n}{\alpha}-1}\right) \\
= & \exp \left(\log \frac{C}{(R-\rho)^{2}} \sum_{j=0}^{m} \frac{1}{p_{j}}+6 \sum_{j=0}^{m} \frac{1}{p_{j}} \log p_{j}+\log 4 \sum_{j=0}^{m} \frac{j+1}{p_{j}}+\Theta^{\frac{n}{2 \alpha}} \sum_{j=0}^{m} p_{j}^{\frac{3 n}{\alpha}-1}\right) \\
\leq & \exp \left(c \log \frac{C}{(R-\rho)^{2}}\left[\sum_{j=0}^{m} \frac{1}{p_{j}}+\sum_{j=0}^{m} \frac{1}{p_{j}} \log p_{j}+\sum_{j=0}^{m} \frac{j+1}{p_{j}}\right]+\Theta^{\frac{n}{2 \alpha}} \sum_{j=0}^{m} p_{j}^{\frac{3 n}{\alpha}-1}\right) .
\end{aligned}
$$

Taking in account the definition of $p_{j}$, one can easily prove that

$$
\left[\sum_{j=0}^{m} \frac{1}{p_{j}}+\sum_{j=0}^{m} \frac{1}{p_{j}} \log p_{j}+\sum_{j=0}^{m} \frac{j+1}{p_{j}}\right] \leq c(\sigma) \sum_{j=0}^{+\infty}\left(\frac{2}{2^{*}}\right)^{j \sigma}<c(n),
$$

for some $\sigma \in(0,1)$ and

$$
\sum_{j=0}^{m} p_{j}^{\frac{3 n}{\alpha}-1} \leqslant \sum_{j=0}^{+\infty} p_{j}^{\frac{3 n}{\alpha}-1}=c(n, \alpha)
$$

since by assumption $\frac{3 n}{\alpha}-1<0$.

Therefore,

$$
\left(\int_{B_{\rho}}(1+P)^{p_{m+1}} d x\right)^{\frac{1}{p_{m+1}}} \leqslant \widehat{C}\left(\int_{B_{R}}(1+P)^{p} d x\right)^{\frac{1}{p}},
$$


for every $m \in \mathbb{N}$. Now, letting $m \rightarrow \infty$ we end up with

$$
\sup _{B_{\rho}}|D u| \leq \lim _{m \rightarrow \infty}\left(\int_{B_{\rho}}(1+P)^{p_{m}}\right)^{\frac{1}{p_{m}}} \leq \widehat{C}\left(\int_{B_{R}}(1+|D u|)^{p}\right)^{\frac{1}{p}},
$$

that gives (3.3).

For further needs we observe that the constant $\widehat{C}$ has the form

$$
\widehat{C}=c(n, p, \alpha, \rho, R) \exp \left(c\left(n, N, p, L_{1}, \nu, \alpha\right)\left(\|k\|_{L^{n} \log ^{\alpha} L}+\|f\|_{L^{n} \log ^{\alpha} L}\right)^{\vartheta}\right)
$$

where $\vartheta=\vartheta(n)$.

Step 4. In this Step we are going to establish estimate (3.4). To this aim it suffices to write estimate (3.5) with $\gamma=0$ and with $\eta$ a cut off function between concentric balls $B_{\rho}$ and $B_{R}$, to get

$$
\begin{aligned}
\int_{B_{\rho}} \Phi(P)|D u|^{p-2}\left|D^{2} u\right|^{2} d x & \leq C \int_{B_{R}} k^{2}|D u|^{p} d x \\
& +\frac{C}{(R-\rho)^{2}} \int_{B_{R}}|D u|^{p} d x+C \int_{B_{R}}|f|^{2} d x .
\end{aligned}
$$

Using estimate (3.3), we obtain

$$
\begin{aligned}
\int_{B_{\rho}} \Phi(P)|D u|^{p-2}\left|D^{2} u\right|^{2} d x & \leq C \sup _{B_{R}}|D u|^{p} \int_{B_{R}} k^{2} d x+\frac{C\left|B_{R}\right|}{(R-\rho)^{2}} \sup _{B_{R}}|D u|^{p}+C \int_{B_{R}}|f|^{2} d x \\
& \leq \hat{C}\left(\int_{B_{2 R}}(1+|D u|)^{p} d x\right),
\end{aligned}
$$

for a constant $\hat{C}=\hat{C}\left(n, N, p, L, L_{1}, \nu, \rho, R,\|k\|_{L^{n} \log ^{\alpha} L\left(B_{2 R}\right)},\|f\|_{L^{n} \log ^{\alpha} L\left(B_{2 R}\right)}\right)$.

The proof is finished in case $n>2$. In the case $n=2$, it suffices to use the appropriate MoserTrudinger Sobolev inequality and argue in the same way.

\section{Proof of Theorem 1}

This section is devoted to the proof of Theorem 1. To this end, we state first an approximation result, which we take from [5, 11. It shows that one can find a sequence of uniformly elliptic integrands $\mathcal{F}_{m}$ that approximate the given $\mathcal{F}$. The approximants can be chosen to be smooth in the $x$ variable, and also to have ellipticity bounds on the whole $\mathbb{R}^{n \times N}$, although these bounds may depend on $m$ (see conditions $\left(\tilde{\mathbf{F}}_{\mathbf{m}} \mathbf{2}\right)-\left(\tilde{\mathbf{F}}_{\mathbf{m}} \mathbf{4}\right)$ below). Furthermore, these ellipticity conditions may be assumed uniform in $m$ away from a ball of the $\xi$ variable (see conditions $(\tilde{\mathbf{F}} \mathbf{0})-(\tilde{\mathbf{F}} \mathbf{4})$ below). We recall that, without loss of generality, we assumed that the radius $\mathcal{R}$ appearing in the assumptions (F0)-(F4) is equal to 1.

Proposition 6. Let $\mathcal{F}: \Omega \times \mathbb{R}^{n \times N} \rightarrow[0,+\infty)$ be a Carathéodory function, convex with respect to the second variable, and satisfying assumptions $(\mathbf{F 0})-(\mathbf{F 4})$. For a fixed open set $\Omega^{\prime} \Subset \Omega$, there exists a sequence $\mathcal{F}_{m}: \Omega^{\prime} \times \mathbb{R}^{n \times N} \rightarrow[0,+\infty)$ of Carathéodory functions, $C^{2}$ and convex in the second variable, such that $\mathcal{F}_{m}$ converges to $\mathcal{F}$ pointwise a.e. on $\Omega^{\prime}$ and everywhere in $\mathbb{R}^{n \times N}$. Moreover, each $\mathcal{F}_{m}$ can be chosen so that the following properties are satisfied: 


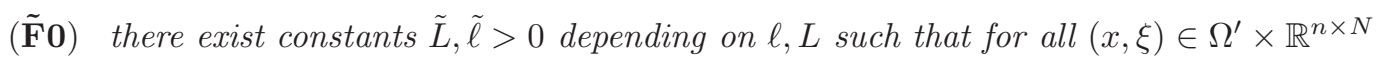

$$
\tilde{\ell}\left(|\xi|^{p}-1\right) \leq \mathcal{F}_{m}(x, \xi) \leq \tilde{L}(1+|\xi|)^{p},
$$

$(\tilde{\mathbf{F}} \mathbf{1})$ for every $x \in \Omega^{\prime}$ and $\xi \in \mathbb{R}^{n N} \backslash B_{2}(0)$ one has $\mathcal{F}_{m}(x, \xi)=F_{m}(x,|\xi|)$,

$(\tilde{\mathbf{F}} \mathbf{2})$ there exists $\tilde{\nu}=\tilde{\nu}(\nu, p)$ such that for every $x \in \Omega^{\prime}, \xi \in \mathbb{R}^{n \times N} \backslash B_{2}(0)$ and $\lambda \in \mathbb{R}^{n \times N}$

$$
\tilde{\nu}(1+|\xi|)^{p-2}|\lambda|^{2} \leq\left\langle D_{\xi \xi} \mathcal{F}_{m}(x, \xi) \lambda, \lambda\right\rangle,
$$

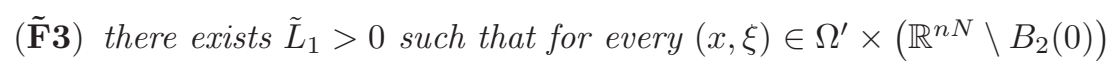

$$
\left|D_{\xi \xi} \mathcal{F}_{m}(x, \xi)\right| \leq \tilde{L}_{1}(1+|\xi|)^{p-2},
$$

( $\tilde{\mathbf{F}} 4)$ for every $x \in \Omega^{\prime}$ and $\xi \in \mathbb{R}^{n N} \backslash B_{2}(0)$,

$$
\left|D_{\xi x} \mathcal{F}_{m}(x, \xi)\right| \leq 2^{p-1} k_{m}(x)(1+|\xi|)^{p-1}
$$

where $k_{m} \in C^{\infty}\left(\Omega^{\prime}\right)$ is a non-negative function such that $k_{m} \rightarrow k$ strongly in $L^{n} \log ^{\alpha} L\left(\Omega^{\prime}\right)$.

Moreover, the above properties can be extended to every $\xi \in \mathbb{R}^{n \times N}$ in the following way:

$\left(\tilde{\mathbf{F}}_{\mathbf{m}} \mathbf{2}\right)$ There exists $\mu_{m}>0$ such that for every $(x, \xi) \in \Omega^{\prime} \times \mathbb{R}^{n \times N}$ and for every $\lambda \in \mathbb{R}^{n \times N}$

$$
\mu_{m}(1+|\xi|)^{p-2}|\lambda|^{2} \leq\left\langle D_{\xi \xi} \mathcal{F}_{m}(x, \xi) \lambda, \lambda\right\rangle,
$$

$\left(\tilde{\mathbf{F}}_{\mathbf{m}} \mathbf{3}\right)$ there exists $\gamma_{m}>0$ such that for every $(x, \xi) \in \Omega^{\prime} \times \mathbb{R}^{n N}$

$$
\left|D_{\xi \xi} \mathcal{F}_{m}(x, \xi)\right| \leq \gamma_{m}(1+|\xi|)^{p-2},
$$

$\left(\tilde{\mathbf{F}}_{\mathbf{m}} \mathbf{4}\right)$ there exists $\Lambda_{m}>0$ such that for every $x \in \Omega^{\prime}$ and $\xi \in \overline{B_{2}(0)}$

$$
\left|D_{\xi x} \mathcal{F}_{m}(x, \xi)\right| \leq \Lambda_{m}(1+|\xi|)^{p-1} .
$$

We now recall a regularity result for minimizers of functionals of the form

$$
\inf _{w} \int_{\Omega}\left(\mathcal{F}(x, D w)+\arctan \left(|w-\bar{u}|^{2}\right)\right) d x
$$

where $\mathcal{F}$ has standard growth conditions and smooth dependence on the $x$-variable, and $\bar{u}$ is fixed. In absence of the perturbation term $\arctan \left(|w-\bar{u}|^{2}\right)$, this regularity result is well known. We refer to [25] for the higher differentiability result, and to [11, Theorem 1.1] as far as the Lipschitz continuity of the local minimizers is concerned. In presence of the perturbation term, the proofs can be easily adapted because of the boundedness of the function $\arctan \left(|w-\bar{u}|^{2}\right)$ and of its derivative with respect to the variable $w$, thus obtaining the following

Theorem 7. Let $\mathcal{G}: \Omega \times \mathbb{R}^{n \times N} \rightarrow[0,+\infty), \mathcal{G} \in C^{2}\left(\Omega \times \mathbb{R}^{n \times N}\right)$, and define the functional

$$
\int_{\Omega}\left(\mathcal{G}(x, D w)+\arctan \left(|w-\bar{u}|^{2}\right)\right) d x
$$


with $\bar{u} \in C^{2}\left(\Omega ; \mathbb{R}^{N}\right)$. Assume that there exists $p \geq 2$ such that for every $x \in \Omega$ and every $\xi, \lambda \in$ $\mathbb{R}^{n \times N}$,

$$
\begin{gathered}
c_{1}|\xi|^{p}-c_{2} \leq \mathcal{G}(x, \xi) \leq L(1+|\xi|)^{p}, \\
\nu(1+|\xi|)^{p-2}|\lambda|^{2} \leq\left\langle D_{\xi \xi} \mathcal{G}(x, \xi) \lambda, \lambda\right\rangle, \\
\left|D_{\xi \xi} \mathcal{G}(x, \xi)\right| \leq L_{1}(1+|\xi|)^{p-2}, \\
\left|D_{\xi x} \mathcal{G}(x, \xi)\right| \leq K(1+|\xi|)^{p-1},
\end{gathered}
$$

with positive constants $c_{1}, c_{2}, L, L_{1}, \nu, K$. Then any local minimizer $v \in W_{\text {loc }}^{1, p}(\Omega)$ of (4.1) is in $W_{\mathrm{loc}}^{2,2}\left(\Omega ; \mathbb{R}^{N}\right)$ and

$$
\left(1+|D v|^{2}\right)^{\frac{p-2}{2}}\left|D^{2} v\right|^{2} \in L_{l o c}^{1}(\Omega) .
$$

Moreover, if there exists $G: \Omega \times[0,+\infty) \rightarrow[0,+\infty)$ such that $\mathcal{G}(x, \xi)=G(x,|\xi|)$, then one also has $v \in W_{\text {loc }}^{1, \infty}\left(\Omega ; \mathbb{R}^{N}\right)$.

We are now ready for proving Theorem 1 .

Proof of Theorem [1. Let $u \in W_{\text {loc }}^{1, p}(\Omega)$ be a local minimizer of the functional $\mathbb{F}(u, \Omega)$, and let $B_{r} \subset \Omega$ be a fixed ball. We consider the sequence of energy densities $\mathcal{F}_{m}(x, \xi)$ obtained after applying Proposition 6 to the integrand $\mathcal{F}$. For a standard sequence of mollifiers $\rho_{\varepsilon}$, we set $u_{\varepsilon}=u * \rho_{\varepsilon}$, $f_{\varepsilon}=f * \rho_{\varepsilon}$ and define

$$
\mathbb{F}_{\varepsilon, m}\left(w, B_{r}\right):=\int_{B_{r}}\left(\mathcal{F}_{m}(x, D w)+f_{\varepsilon}(x) w+\arctan \left|w-u_{\varepsilon}\right|^{2}\right) d x .
$$

The lower semi-continuity and strict convexity of $\mathbb{F}_{\varepsilon, m}$ with respect to the gradient variable ensure that the minimization problem

$$
\min \left\{\mathbb{F}_{\varepsilon, m}\left(w ; B_{r}\right): w \in u+W_{0}^{1, p}\left(B_{r}, \mathbb{R}^{N}\right)\right\}
$$

has a unique solution $v_{\varepsilon, m} \in u+W_{0}^{1, p}\left(B_{r}, \mathbb{R}^{N}\right)$. By the growth conditions of $\mathcal{F}_{m}$ stated in $(\tilde{\mathbf{F}} \mathbf{0})$ of Proposition 6 and the minimality of $v_{\varepsilon, m}$, we deduce that

$$
\begin{aligned}
\tilde{\ell} \int_{B_{r}}\left|D v_{\varepsilon, m}\right|^{p} d x & \leq \int_{B_{r}}\left(\tilde{\ell}+\mathcal{F}_{m}\left(x, D v_{\varepsilon, m}\right)\right) d x \\
& \leq \mathbb{F}_{\varepsilon, m}\left(v_{\varepsilon, m}, B_{r}\right)+\int_{B_{r}}\left(\tilde{\ell}-f_{\varepsilon}(x) \cdot v_{\varepsilon, m}\right) d x \\
& \leq \mathbb{F}_{\varepsilon, m}\left(u, B_{r}\right)+\int_{B_{r}}\left(\tilde{\ell}-f_{\varepsilon}(x) \cdot v_{\varepsilon, m}\right) d x \\
& =\int_{B_{r}}\left(\mathcal{F}_{m}(x, D u)+\arctan \left|u-u_{\varepsilon}\right|^{2}\right) d x-\int_{B_{r}} f_{\varepsilon}(x)\left(v_{\varepsilon, m}-u\right) d x+\tilde{\ell}\left|B_{r}\right| \\
& \leq \int_{B_{r}}\left(\tilde{L}|D u|^{p}+\frac{\pi}{2}\right) d x+\int_{B_{r}}\left|f_{\varepsilon}(x)\right|\left|v_{\varepsilon, m}-u\right| d x+(\tilde{\ell}+\tilde{L})\left|B_{r}\right| .
\end{aligned}
$$

Hence

$$
\int_{B_{r}}\left|D v_{\varepsilon, m}\right|^{p} d x \leq C \int_{B_{r}}|D u|^{p} d x+\int_{B_{r}}\left|f_{\varepsilon}(x)\right|\left|v_{\varepsilon, m}-u\right| d x+C\left|B_{r}\right|
$$


with a constant $C=C(\tilde{\ell}, \tilde{L})$ independent of $m$ and of $\varepsilon$.

Using Young's and Poincaré inequalities in the last integral of previous estimate, we get

$$
\begin{aligned}
\int_{B_{r}} & \left|D v_{\varepsilon, m}\right|^{p} d x \leq C \int_{B_{r}}\left(1+|D u|^{p}\right) d x+c_{\kappa} \int_{B_{r}}\left|f_{\varepsilon}(x)\right|^{p^{\prime}} d x+\kappa \int_{B_{r}}\left|v_{\varepsilon, m}-u\right|^{p} d x \\
& \leq C \int_{B_{r}}\left(1+|D u|^{p}\right) d x+c_{\kappa} \int_{B_{r}}\left|f_{\varepsilon}(x)\right|^{p^{\prime}} d x+\kappa c_{n, p, r} \int_{B_{r}}\left|D v_{\varepsilon, m}-D u\right|^{p} d x, \\
& \leq C \int_{B_{r}}\left(1+|D u|^{p}\right) d x+c_{\kappa} \int_{B_{r}}\left|f_{\varepsilon}(x)\right|^{p^{\prime}} d x+\kappa c_{n, p, r} \int_{B_{r}}|D u|^{p} d x+\kappa c_{n, p, r} \int_{B_{r}}\left|D v_{\varepsilon, m}\right|^{p} d x,
\end{aligned}
$$

Choosing $\kappa=\frac{1}{2 c_{n, p, r}}$, we can reabsorb the last integral in the right hand side of previous inequality by the left hand side, getting

$$
\int_{B_{r}}\left|D v_{\varepsilon, m}\right|^{p} d x \leq C \int_{B_{r}}\left(1+|D u|^{p}\right) d x+C \int_{B_{r}}\left|f_{\varepsilon}(x)\right|^{p^{\prime}} d x
$$

for a constant $C$ independent of $\varepsilon$ and $m$ and so with right hand side independent of $m$. Moreover, since $p^{\prime} \leq 2 \leq n$, the strong convergence of $f_{\varepsilon}$ to $f$ in $L^{n} \log ^{\alpha} L$, implies that

$$
\int_{B_{r}}\left|f_{\varepsilon}(x)\right|^{p^{\prime}} d x \leqslant C
$$

with $C$ independent of $\varepsilon$. Therefore

$$
\int_{B_{r}}\left|D v_{\varepsilon, m}\right|^{p} d x \leq C
$$

with a constant $C$ independent of $\varepsilon$ and $m$. Hence, by weak compactness, we deduce that there exists $v_{\varepsilon} \in u+W_{0}^{1, p}\left(B_{r} ; \mathbb{R}^{N}\right)$ such that

$$
\left\{v_{\varepsilon, m}\right\}_{m} \rightarrow v_{\varepsilon} \quad \text { weakly in } W^{1, p}\left(B_{r}\right)
$$

and

$$
\left\{v_{\varepsilon, m}\right\}_{m} \rightarrow v_{\varepsilon} \quad \text { strongly in } L^{p}\left(B_{r}\right)
$$

as $m \rightarrow+\infty$, up to a subsequence. Set now

$$
\mathbb{F}_{\varepsilon}\left(w, B_{r}\right):=\int_{B_{r}}\left(\mathcal{F}(x, D w)+f_{\varepsilon}(x) w+\arctan \left(\left|w-u_{\varepsilon}\right|^{2}\right)\right) d x .
$$

For every fixed $\varepsilon>0$, one can see that the functionals $\mathbb{F}_{\varepsilon, m} \Gamma$-converge to $\mathbb{F}_{\varepsilon}$ as $m \rightarrow \infty$ (see Theorem 5.14 and Corollary 7.20 in [12]). As a consequence, $v_{\varepsilon}$ is a minimizer of $\mathbb{F}_{\varepsilon}$. Now, the weak lower semicontinuity of the $L^{p}$ norm and (4.3) imply that

$$
\int_{B_{r}}\left|D v_{\varepsilon}\right|^{p} d x \leq \liminf _{m \rightarrow \infty} \int_{B_{r}}\left|D v_{\varepsilon, m}\right|^{p} d x \leqslant C .
$$

Since the constant $C$ in (4.6) is independent of $\varepsilon$, as before, by compactness there exists $v \in$ $u+W_{0}^{1, p}\left(B_{r} ; \mathbb{R}^{N}\right)$ such that

$$
\left\{v_{\varepsilon}\right\}_{\varepsilon} \rightarrow v \quad \text { weakly in } W^{1, p}\left(B_{r}\right)
$$

and

$$
\left\{v_{\varepsilon}\right\}_{\varepsilon} \rightarrow v \quad \text { strongly in } L^{p}\left(B_{r}\right)
$$


as $\varepsilon \rightarrow 0$, up to a subsequence. Also, again by the weak lower semicontinuity of the norm,

$$
\int_{B_{r}}|D v|^{p} d x \leq \liminf _{\varepsilon \rightarrow 0} \int_{B_{r}}\left|D v_{\varepsilon}\right|^{p} d x \leqslant C .
$$

Observe that, as $\varepsilon \rightarrow 0$, the functionals $\mathbb{F}_{\varepsilon} \Gamma$-converge to

$$
\mathbb{F}_{0}\left(w, B_{r}\right):=\int_{B_{r}}\left(\mathcal{F}(x, D w)+f(x) \cdot w+\arctan \left(|w-u|^{2}\right)\right) d x
$$

whence $v$ is a minimizer of $\mathbb{F}_{0}$ coinciding with $u$ on $\partial B_{r}$, and therefore $\mathbb{F}_{0}\left(v, B_{r}\right) \leq \mathbb{F}_{0}\left(u, B_{r}\right)$. This, together with the minimality of $u$, implies that

$$
\mathbb{F}\left(u, B_{r}\right) \leq \mathbb{F}\left(v, B_{r}\right) \leq \mathbb{F}_{0}\left(v, B_{r}\right) \leq \mathbb{F}_{0}\left(u, B_{r}\right)=\mathbb{F}\left(u, B_{r}\right)
$$

Hence all the above inequalities hold true as equalities, and as a consequence

$$
\int_{B_{r}} \arctan |u-v|^{2} d x=0, \quad \Rightarrow \quad u=v \quad \text { a.e. in } B_{r} .
$$

Since the functionals $\mathbb{F}_{\varepsilon, m}$ satisfy the assumptions of Theorem 7 for every $\varepsilon$ and $m$, their minimizers $v_{\varepsilon, m}$ belong to $W_{\text {loc }}^{2,2} \cap W^{1, \infty}$. Therefore we are legitimate to use the a priori estimate at (3.1) of Theorem 4 thus getting

$$
\sup _{B_{\rho}}\left|D v_{\varepsilon, m}\right| \leqslant C \exp \left(C\left(|| k_{\varepsilon}\left\|_{L^{n} \log ^{\alpha} L}+|| f_{\varepsilon}\right\|_{L^{n} \log ^{\alpha} L}\right)^{\vartheta}\right)\left(\int_{B_{\rho^{\prime}}}\left(1+\left|D v_{\varepsilon, m}\right|^{p}\right) d x\right)^{\frac{1}{p}},
$$

for every $B_{\rho} \subset B_{\rho^{\prime}} \Subset B_{r}$ and for a constant $C$ independent of $\varepsilon$ and $m$ by the expression at (3.39). By virtue of (4.6) and (4.9), and since $k_{\varepsilon} \rightarrow k$ and $f_{\varepsilon} \rightarrow f$ strongly in $L^{n} \log ^{\alpha} L$, passing to the limit, first as $m \rightarrow+\infty$, and then as $\varepsilon \rightarrow 0$ in estimate (4.10), we conclude that

$$
\sup _{B_{\rho}}|D u| \leqslant C \exp \left(C\left(|| k\left\|_{L^{n} \log ^{\alpha} L}+\right\| f \|_{L^{n} \log ^{\alpha} L}\right)^{\vartheta}\right)\left(\int_{B_{\rho^{\prime}}}\left(1+|D u|^{p}\right) d x\right) .
$$

This finishes the proof.

\section{Proof of Theorem 2}

In this section, we establish the integrability of second order distributional derivatives of the local minimizers of the functional $\mathbb{F}(\cdot, \Omega)$. To this aim, we will need a measure theory tool, that is inspired by a result in [9]. The result we present here goes a bit further, as it states convergence on the set $\{|D f|>1\}$, and not only on $\{|D f|>t\}$ for each $t>1$ and this improvement is due to the Lipschitz regularity of the minimizers proven in Theorem [1. The precise statement is as follows.

Proposition 8. Let $p \geq 2, N \geq 1$, and let $f_{k}, f \in W^{1, p}\left(\Omega ; \mathbb{R}^{N}\right)$ be given, and denote $P_{k}=$ $\left(\left|D f_{k}\right|-1\right)_{+}$. Assume that:

(a) $f_{k} \rightarrow f$ in $W^{1, p}\left(\Omega ; \mathbb{R}^{N}\right)$,

(b) $P_{k} \in L^{\infty}$ and there exists a positive constant $M$ independent of $k$ such that

$$
\left\|P_{k}\right\|_{L^{\infty}(\Omega)} \leq M
$$

for every $k \in \mathbb{N}$. 
(c) Assume that there exists a positive constant $N$ independent of $k$ such that

$$
\int_{\Omega} \frac{P_{k}^{p}}{\left(1+P_{k}\right)^{2}}\left|D P_{k}\right|^{2} d x \leq N
$$

for every $k \in \mathbb{N}$.

Then one has $(|D f|-1)_{+}^{\frac{p}{2}+1} \in W^{1,2}(\Omega)$. Moreover, there exists a not relabeled subsequence $f_{k}$ such that

$$
\lim _{k \rightarrow \infty}\left|D f_{k}\right|=|D f| \quad \text { strongly in } L^{p+2}(\Omega \cap\{|D f|>1\})
$$

and

$$
\lim _{k \rightarrow \infty}\left|D f_{k}\right|=|D f| \quad \text { a.e. in } \Omega \cap\{|D f|>1\} .
$$

Proof. First, it is immediate to see that

$$
\begin{aligned}
\int_{\Omega}\left|D\left(P_{k}^{\frac{p+2}{2}}\right)\right|^{2} & =c(p) \int_{\Omega} P_{k}^{p}\left|D P_{k}\right|^{2}=c(p) \int_{\Omega} \frac{P_{k}^{p}}{\left(1+P_{k}\right)^{2}}\left(1+P_{k}\right)^{2}\left|D P_{k}\right|^{2} \\
& \leq c(p, M) \int_{\Omega} \frac{P_{k}^{p}}{\left(1+P_{k}\right)^{2}}\left|D P_{k}\right|^{2} \leq c(p, M, N),
\end{aligned}
$$

where we used assumptions $(b)$ and $(c)$. By compactness, there exists $\varphi \in W^{1,2}(\Omega)$ such that $P_{k}^{\frac{p+2}{2}} \rightarrow \varphi$ weakly in $W^{1,2}(\Omega)$ and strongly in $L^{2}(\Omega)$. As a consequence, $\varphi \geq 0$ almost everywhere. Using also that $r \mapsto r^{\frac{2}{2+p}}$ is $\frac{2}{2+p}$-Hölder continuous on $[0, \infty)$, we can deduce that

$$
\int_{\Omega}\left|P_{k}-\varphi^{\frac{2}{p+2}}\right|^{p+2} \leq c(p) \int_{\Omega}\left|P_{k}^{\frac{p+2}{2}}-\varphi\right|^{2}
$$

and therefore $P_{k} \rightarrow \varphi^{\frac{2}{p+2}}$ strongly in $L^{p+2}(\Omega)$ and also in measure. From now on, let us denote $P=\varphi^{\frac{2}{p+2}}$ and, recalling the definition of $P_{k}$, observe that

$$
\begin{aligned}
& \lim _{k \rightarrow+\infty} \int_{\Omega}\left|P_{k}-P\right|^{p+2} \\
= & \lim _{k \rightarrow+\infty} \int_{\left\{x \in \Omega:\left|D f_{k}\right| \leq 1\right\}}|P|^{p+2}+\lim _{k \rightarrow+\infty} \int_{\left\{x \in \Omega:\left|D f_{k}\right|>1\right\}}|| D f_{k}|-1-P|^{p+2}=0
\end{aligned}
$$

that, in particular, implies

$$
\lim _{k \rightarrow+\infty} \int_{\left\{x \in \Omega:\left|D f_{k}\right|>1\right\}}|| D f_{k}|-1-P|^{p+2}=0 .
$$

Moreover by the convergence in measure of $P_{k}$ to $P$, for every $t>0$, we have

$$
\begin{aligned}
0 & =\lim _{k \rightarrow+\infty}\left|\left\{x \in \Omega:\left|\left(\left|D f_{k}\right|-1\right)_{+}-P\right| \geq t\right\}\right| \\
& =\lim _{k \rightarrow+\infty} \mid\left\{x \in \Omega:\left|D f_{k}\right| \leq 1 \text { and }|P| \geq t\right\} \mid \\
& +\lim _{k \rightarrow+\infty} \mid\left\{x \in \Omega:\left|D f_{k}\right|>1 \text { and }|| D f_{k}|-1-P| \geq t\right\} \mid
\end{aligned}
$$

that, in particular, yields

$$
0=\lim _{k \rightarrow+\infty} \mid\left\{x \in \Omega:\left|D f_{k}\right| \leq 1 \text { and }|P| \geq t\right\} \mid
$$


For $t>0$, we write

$$
\begin{aligned}
& \int_{\{x \in \Omega: P \geq t\}}|| D f_{k}|-1-P|^{p+2} \\
= & \int_{\left\{\left|D f_{k}\right| \leq 1 \text { and } P \geq t\right\}}|| D f_{k}|-1-P|^{p+2}+\int_{\left\{\left|D f_{k}\right|>1 \text { and } P \geq t\right\}}|| D f_{k}|-1-P|^{p+2} \\
= & I_{1, k}+I_{2, k} .
\end{aligned}
$$

By (5.3) we have that

$$
\lim _{k \rightarrow+\infty} I_{2, k} \leq \lim _{k \rightarrow+\infty} \int_{\left\{\left|D f_{k}\right|>1\right\}}|| D f_{k}|-1-P|^{p+2}=0 .
$$

Since by virtue of the assumption $(b)$, we have $\|P\|_{\infty} \leq c(M)$, we get

$$
\begin{aligned}
\lim _{k \rightarrow+\infty} I_{1, k} & \leq c(p) \lim _{k \rightarrow+\infty} \int_{\left\{\left|D f_{k}\right| \leq 1 \text { and } P \geq t\right\}}(2+|P|)^{p+2} \\
& \leq c(M, p) \lim _{k \rightarrow+\infty} \mid\left\{\left|D f_{k}\right| \leq 1 \text { and } P \geq t\right\} \mid=0,
\end{aligned}
$$

by the equality in (5.4). Inserting (5.7) and (5.6) in (5.5), we conclude

$$
\lim _{k \rightarrow+\infty} \int_{\{P \geq t\}}|| D f_{k}|-1-P|^{p+2}=0
$$

for every $t>0$. One can easily observe that

$$
B(0):=\{x \in \Omega: P>0\}=\bigcup_{n \in \mathbb{N}}\left\{x \in \Omega: P \geq \frac{1}{n}\right\}=: \bigcup_{n \in \mathbb{N}} B\left(\frac{1}{n}\right)
$$

and, since $B\left(\frac{1}{n}\right) \subset B\left(\frac{1}{n+1}\right)$,

$$
|\{x \in \Omega: P>0\}|=\lim _{n \rightarrow+\infty}\left|\left\{x \in \Omega: P \geq \frac{1}{n}\right\}\right| .
$$

Since $B\left(\frac{1}{n}\right) \subset B(0)$, for every $n \in \mathbb{N}$,

$$
\left\|\chi_{B(0)}-\chi_{B(1 / n)}\right\|_{L^{1}(\Omega)}=\left\|\chi_{B(0) \backslash B(1 / n)}\right\|_{L^{1}(\Omega)}=|B(0)-B(1 / n)| \rightarrow 0 .
$$

Therefore

$$
\begin{aligned}
& \int_{\{P>0\}}|| D f_{k}|-1-P|^{p+2} \\
= & \int_{\{P>0\}}|| D f_{k}|-1-P|^{p+2}-\int_{\{P \geq 1 / n\}}|| D f_{k}|-1-P|^{p+2} \\
+ & \int_{\{P \geq 1 / n\}}|| D f_{k}|-1-P|^{p+2} \\
= & \int_{\left|D f_{k}\right|-1-\left.P\right|^{p+2}\left(\chi_{B(0)}-\chi_{B(1 / n)}\right)} \\
+ & \int_{\{P \geq 1 / n\}}|| D f_{k}|-1-P|^{p+2}
\end{aligned}
$$




$$
\leq C(M)\left\|\chi_{B(0)}-\chi_{B(1 / n)}\right\|_{L^{1}(\Omega)}+\int_{\{P \geq 1 / n\}}|| D f_{k}|-1-P|^{p+2},
$$

where we used assumption (b). Passing to the limit first as $k \rightarrow \infty$ and using (5.8) with $t=1 / n$, and then as $n \rightarrow \infty$ in (5.9), we conclude that

$$
\lim _{k \rightarrow \infty} \int_{\{P>0\}}|| D f_{k}|-1-P|^{p+2}=0 .
$$

From previous equality we deduce that

$$
\left|D f_{k}\right| \rightarrow P+1 \quad \text { strongly in } L^{p+2}(\{P>0\})
$$

and, of course, modulo subsequences, also weakly and almost everywhere. By assumption, $\left(f_{k}\right)$ is weakly convergent in $W^{1, p}\left(\Omega ; \mathbb{R}^{N}\right)$ to $f$, so, by the essential uniqueness of the weak limit we conclude that

$$
|D f|=P+1 \quad \text { a.e. in }\{P>0\} .
$$

Therefore

$$
\{P>0\}=\{x \in \Omega:|D f|>1\} .
$$

By (5.11) and (5.12) and the equality above, there exists a subsequence (not relabeled) of $f_{k}$ such that

$$
\left|D f_{k}\right| \rightarrow_{k \rightarrow \infty}|D f| \quad \text { strongly in } L^{p+2}(\{x \in \Omega:|D f|>1\}) .
$$

The fact that $(|D f|-1)_{+}^{\frac{p}{2}+1} \in W^{1,2}(\Omega)$ now easily follows by the semicontinuity of the $L^{2}$ norm, assumption (5.2) and (5.12).

We can now proceed with the proof of Theorem 2 ,

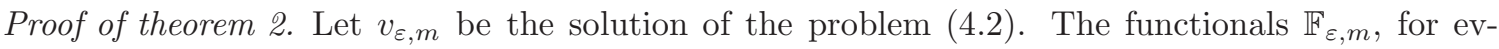
ery $\varepsilon$ and $m$, satisfy the assumptions of Theorem 7 . Therefore their minimizers $v_{\varepsilon, m}$ belong to $W_{\text {loc }}^{2,2} \cap W^{1, \infty}$ and are such that $\left|D v_{\epsilon, m}\right|^{p-2}\left|D^{2} v_{\varepsilon, m}\right|^{2} \in L^{1}$. As a consequence, we are legitimate to use the a priori estimate (3.2) of Theorem 4, thus getting

$$
\int_{B_{\rho}\left(x_{0}\right)} \frac{P_{\varepsilon, m}^{2}}{\left(1+P_{\varepsilon, m}\right)^{2}}\left|D v_{\varepsilon, m}\right|^{p-2}\left|D^{2} v_{\varepsilon, m}\right|^{2} d x \leq C \int_{B_{2 R}\left(x_{0}\right)}\left(1+\left|D v_{\varepsilon, m}\right|^{p}\right) d x,
$$

with constant $\hat{C}=C \exp \left(C\left(\left\|k_{\varepsilon}\right\|_{L^{n} \log ^{\alpha} L_{\mathrm{loc}}(\Omega)}+\left\|f_{\varepsilon}\right\|_{L^{n} \log ^{\alpha} L_{\mathrm{loc}}(\Omega)}\right)^{\vartheta}\right)$ independent of $m$. As usual, $P_{\varepsilon, m}=\left(\left|D v_{\varepsilon, m}\right|-1\right)_{+}$. Now combining estimates (5.14) and (4.3), we obtain

$$
\int_{B_{\rho}\left(x_{0}\right)}\left|D\left(\mathcal{G}\left(P_{\varepsilon, m}\right)\right)\right|^{2} d x=\int_{B_{\rho}\left(x_{0}\right)} \frac{P_{\varepsilon, m}^{2}}{\left(1+P_{\varepsilon, m}\right)^{2}}\left|D v_{\varepsilon, m}\right|^{p-2}\left|D^{2} v_{\varepsilon, m}\right|^{2} \mathrm{~d} x \leq C
$$

with a constant $C$ independent of $m$ and of $\varepsilon$, and where $\mathcal{G}$ is the function defined in (1.2). Furthermore, by estimates (3.1) and (4.3), we have that

$$
\left\|P_{\varepsilon, m}\right\|_{L^{\infty}\left(B_{\rho}\right)} \leq \sup _{B_{\rho}}\left|D v_{\varepsilon, m}\right| \leq C
$$


with a constant $C$ independent of $m$ and $\varepsilon$. Thus, recalling that $v_{\varepsilon, m}$ weakly converges to $v_{\varepsilon}$ in $W^{1, p}\left(B_{r}\right)$, we are legitimate to apply Proposition 8 to the sequence $\left(P_{\varepsilon, m}\right)_{m}$ and this yields

$$
\left|D v_{\varepsilon, m}\right| \rightarrow\left|D v_{\varepsilon}\right| \quad \text { strongly in } L^{p}\left(B_{\rho} \cap\left\{\left|D v_{\varepsilon}\right|>1\right\}\right),
$$

and

$$
\left|D v_{\varepsilon, m}\right| \rightarrow\left|D v_{\varepsilon}\right| \quad \text { a.e. in } B_{\rho} \cap\left\{\left|D v_{\varepsilon}\right|>1\right\},
$$

as $m \rightarrow \infty$. If we now set $w_{\varepsilon, m}=\mathcal{G}\left(P_{\varepsilon, m}\right)$, then from (5.15) we deduce that (up to a subsequence) one has $w_{\varepsilon, m} \rightarrow w_{\varepsilon}$ as $m \rightarrow \infty$ for some $w_{\varepsilon} \in W^{1,2}\left(B_{\rho}\right)$, with weak convergence in $W^{1,2}\left(B_{\rho}\right)$, strong convergence in $L^{2}\left(B_{\rho}\right)$, and a.e. convergence in $B_{\rho}$. The latter, together with (5.17), implies that $w_{\varepsilon}=\mathcal{G}\left(\left(\left|D v_{\varepsilon}\right|-1\right)_{+}\right)$almost everywhere on $B_{\rho} \cap\left\{\left|D v_{\varepsilon}\right|>1\right\}$. But then the lower semicontinuity of the norm implies that

$$
\int_{B_{\rho}\left(x_{0}\right)}\left|D\left(\mathcal{G}\left(\left(\left|D v_{\varepsilon}\right|-1\right)_{+}\right)\right)\right|^{2} \leq C,
$$

with a constant $C$ independent of $\varepsilon$. We now argue for the sequence $v_{\varepsilon}$ as we did for $v_{\varepsilon, m}$, and the theorem follows .

Acknowledgements. A. Clop and F. Hatami were partially supported by project FP7-607647 of the European Comission. A. Clop is also supported by projects 2017-SGR-395 (Catalan Government) and MTM2016-75390 (Spanish Government). R.Giova and A.Passarelli di Napoli have been partially supported by the Gruppo Nazionale per l'Analisi Matematica, la Probabilità e le loro Applicazioni (GNAMPA) of the Istituto Nazionale di Alta Matematica (INdAM).

R. Giova has been partially supported by Università degli Studi di Napoli Parthenope through the projects "sostegno alla Ricerca individuale" (2015 - 2016 - 2017) and "Sostenibilità, esternalità e uso efficiente delle risorse ambientali" (2017-2019)

\section{References}

[1] R.A. Adams, Sobolev spaces. Academic Press, New York-London 1975, Pure and Applied Mathematics, vol. 65 .

[2] L. Brasco, Global $L^{\infty}$ gradient estimates for solutions to a certain degenerate elliptic equation. Nonlinear Anal. 74 (2011), no. 2, 516-531.

[3] L. Brasco, G. Carlier, F. Santambrogio, Congested traffic dynamics, weak flows and very degenerate elliptic equations. J. Math. Pures Appl. (9) 93 (2010), no. 6, 652-671.

[4] G. Carlier, C. Jimenez, F. Santambrogio, Optimal transportation with traffic congestion and Wardrop equilibria. SIAM J. Control Optim. 47 (2008), no. 3, 1330-1350.

[5] P. Celada, G. Cupini, M. Guidorzi, Existence and regularity of minimizers of nonconvex integrals with p-q growth. ESAIM Control Optim. Calc. Var. 13 (2007), 343-358. 
[6] M. Chipot and L.C. Evans, Linearization at infinity and Lipschitz estimates for certain problems in Calculus of Variations. Proc. Roy. Soc. Edinburgh Sect. A 102 (1986), 291-303.

[7] A. Cianchi, Interpolation of operators and Sobolev embedding theorem in Orlicz spaces. International Conference on Differential Equations (Lisboa, 1995). World Sci. Publ., River Edge, pp. 306-310 (1998)

[8] A. Clop, D. Faraco, J. Mateu, J. Orobitx, X. Zhong, Beltrami equations with coefficient in the Sobolev space $W^{1, p}$. Publicacions Matemtiques 53 (1), 197-230.

[9] G. Cupini, F. Giannetti, R. Giova, A. Passarelli di Napoli, Higher integrability for minimizers of asymptotically convex integrals with discontinuous coefficients. Nonlinear Anal. 154 (2017), $7-24$

[10] G. Cupini, F. Giannetti, R. Giova, A. Passarelli di Napoli, Regularity results for vectorial minimizers of a class of degenerate convex integrals. J. Differential Equations 265 (2018), no. $9,4375-4416$.

[11] G. Cupini, M. Guidorzi, E. Mascolo, Regularity of minimizers of vectorial integrals with $p-q$ growth. Nonlinear Anal. 54 (2003), no. 4, 591-616.

[12] G. Dal Maso, An introduction to $\Gamma$-convergence. Progr. Nonlinear Differential Equations Appl., no. 8, Birkhäuser, Boston, 1993.

[13] M. Eleuteri, P. Marcellini, E. Mascolo, Lipschitz estimates for systems with ellipticity conditions at infinity. Ann. Mat. Pura Appl., 195 (2016), 1575-1603.

[14] M. Eleuteri, P. Marcellini, E. Mascolo, Lipschitz continuity for energy integrals with variable exponents., Rend. Lincei, Matematica E Applicazioni, 27 (2016), no. 1, p. 61

[15] M. Eleuteri, P. Marcellini, E. Mascolo, Regularity for scalar integrals without structure conditions. Adv. Calc. Var., DOI: doi.org/10.1515/acv-2017-0037

[16] I. Fonseca, N.Fusco, P. Marcellini, An existence result for a nonconvex variational problem via regularity. ESAIM Control Optim. Calc. Var. 7 (2002), 69-95.

[17] M. Foss, A. Passarelli di Napoli and A. Verde, Global Morrey regularity results for asymptotically convex variational problems. Forum Math. 20 (2008), 921-953.

[18] M. Foss, A. Passarelli di Napoli and A. Verde, Global Lipschitz regularity for almost minimizers of asymptotically convex variational problems. Ann. Mat. Pura Appl. 189 (2010), no.4, 127162 .

[19] M. Foss, C. S. Goodrich, Partial Hölder continuity of minimizers of functionals satisfying a general asymptotic relatedness condition. J. Convex Anal. 22 (2015), 219-246.

[20] M. Foss, C. S. Goodrich, On partial Hölder continuity and a Caccioppoli inequality for minimizers of asymptotically convex functionals between Riemannian manifolds. Ann. Mat. Pura Appl. 195 (2016),no. 4, 1405-1461. 
[21] C. S. Goodrich, On nonlinear boundary conditions satisfying certain asymptotic behavior. Nonlinear Anal. 76 (2013), 58-67.

[22] C. S. Goodrich, Partial Hölder continuity of minimizers of functionals satisfying a VMO condition. Adv. Calc. Var. 10 (2017), 83-110.

[23] C. S. Goodrich, Partial Lipschitz regularity of minimizers of asymptotically convex functionals with general growth conditions. J. Differential Equations 263 (2017), 4400-4428.

[24] C. S. Goodrich, Partial regularity of minimizers of functionals with discontinuous coefficients of low integrability with applications to nonlinear elliptic systems. Comm. Partial Differential Equations, doi: 10.1080/03605302.2018.1517794

[25] M. Giaquinta, G. Modica, Remarks on the regularity of the minimizers of certain degenerate functionals. Manuscripta Math. 57 (1986) 55-99.

[26] R. Giova, Higher differentiability for n-harmonic systems with Sobolev coefficients. J. Differential Equations 259 (2015), 5667-5687.

[27] R. Giova, Regularity results for non-autonomous functionals with $L \log L$-growth and Orlicz Sobolev coefficients. NoDEA Nonlinear Differential Equations Appl. 23 (2016), no. 6, Art. 64, $18 \mathrm{pp}$.

[28] R. Giova, A. Passarelli di Napoli, Higher differentiability of a priori bounded minimizers of convex variational integrals with discontinuous coefficients - Advances in Calculus of Variations 12 (2019), no. 1, 85-110.

[29] E. Giusti. Direct methods in the calculus of variations. World Scientific, 2003.

[30] M. Kronz, Partial regularity results for minimizers of quasiconvex functionals of higher order. Ann. Inst. H. Poincar Anal. Non Linéaire 19 (2002), no. 1, 81-112.

[31] C. Leone, A. Passarelli di Napoli and A. Verde, Lipschitz regularity for some asymptotically subquadratic problems. Nonlinear Anal. 67 (2007), 1532-1539.

[32] A. Passarelli di Napoli, Higher differentiability of minimizers of variational integrals with Sobolev coefficients. Adv. Calc. Var. 7 (2014), no. 1, 59-89.

[33] A. Passarelli di Napoli, Higher differentiability of solutions of elliptic systems with Sobolev coefficientes: the case $p=n=2$. Potential Anal. 41 (2014), no. 3, 715-735

[34] A. Passarelli di Napoli, Regularity results for non-autonomous variational integrals with discontinuous coefficients. Rend. Lincei Mat. Appl. 26 (2015), no. 4, 475-496

[35] A. Passarelli di Napoli and A. Verde, A regularity result for asymptotically convex problems with lower order terms. J. Convex Anal. 15 (2008), 131-148.

[36] J.P. Raymond, Lipschitz regularity for some asymptotically convex problems. Proc. Roy. Soc. Edinburgh Sect. A 117 (1991), 59-73. 
[37] C. Scheven and T. Schmidt, Asymptotically regular problems. II. Partial Lipschitz continuity and a singular set of positive measure. Ann. Sc. Norm. Super. Pisa Cl. Sci. 8 (2009), no. 5, 469-507.

[38] C. Scheven and T. Schmidt, Asymptotically regular problems. I. Higher Integrability. J. Differential Equations. 248 (2010), 745-791.

\section{A. Clop, F. Hatami}

Departament de Matemàtiques,

Facultat de Ciències, Campus de la U.A.B.

08193-Bellaterra (CATALONIA)

E-mail address: albertcp@mat.uab.cat, fhatami@mat.uab.cat

\section{R. Giova}

Università degli Studi di Napoli "Parthenope"

Palazzo Pacanowsky - Via Generale Parisi, 13

80132 Napoli, Italy

E-mail address: raffaella.giova@uniparthenope.it

\section{A. Passarelli di Napoli}

Università degli Studi di Napoli "Federico II"

Dipartimento di Mat. e Appl. "R. Caccioppoli"

Via Cintia 80126 Napoli, Italy

E-mail address: antpassa@unina.it 